\title{
Abstracts from HSG 2017
}

Published online: 13 November 2017

(C) The American Society for Experimental NeuroTherapeutics, Inc. 2017

\section{Poster \#1}

Current Challenges and Future Opportunities: The Huntington Disease Care Improvement Project

Karen Anderson ${ }^{1}$, Jack Griffin ${ }^{2}$, Stevan Ramirez ${ }^{3}$, Joni Steinman $^{4}, \mathrm{Al} \mathrm{Kinel}^{4}$, Shari Kinel ${ }^{3}$

${ }^{1}$ Georgetown University, Washington, DC, USA

${ }^{2}$ Griffin Foundation, Potomac, MD, USA

${ }^{3}$ Huntington Study Group, Rochester, NY, USA

${ }^{4}$ Strategic Interests, LLC, Rochester, NY, USA

\begin{abstract}
Background: Numerous Huntington disease (HD) advocacy and research groups exist in the USA, but there has been minimal strategic collaboration between them to date. This project investigated challenges faced by key community stakeholders that utilize one or multiple HD groups, and strategized next steps regarding solutions.
\end{abstract}

Objectives: To assess the relationship between various HD advocacy and research organizations, and to strategize future opportunities for the HD community.

Methods: In conjunction with the Huntington Study Group, PriceWaterhouseCoopers $(\mathrm{PwC})$ created a structured guide to facilitate stakeholder interviews with a focus on patientcentered outcomes, multidisciplinary care coordination, and optimizing the HD care experience. PwC conducted 20 detailed interviews with a broad range of stakeholders, including care providers and social workers, basic and clinical researchers, leadership of key HD organizations, and leadership of non-HD organizations (e.g., biopharma).

Results: A number of objectives were defined for collaboration within the HD community: 1) sharing information and data about recent drug approvals and a robust pipeline of HD drug development; 2) enhancing significant reach and credibility of HD advocacy organizations within the HD community and providing a strong potential foundation for new strategic partnerships; 3) addressing untapped potential for convening and catalyzing HD community stakeholders, which limits progress in both clinical care and research.

Conclusions: There is a need for cohesive action by HD organizations. Recommendations: 1) patient engagement and health promotion platform-develop an online community for patients with HD and caregivers to express patient voice, share information, and catalyze partnerships; 2 ) translational research consortium-develop a patientcentered research collaborative enabling patients, researchers, and clinicians to share data and expertise to enhance precision medicine in partnership with payers, funders, and industry; 3 ) integrated community care pilot - pilot multidisciplinary care approaches with funding partners that can advance best practices for care with improved outcomes, efficiencies, and cost savings.

\section{Poster \#2}

Improving Care for People with Huntington's Disease and Their Families: The Huntington Disease Care Improvement Project

Karen Anderson ${ }^{1}$, Jack Griffin ${ }^{2}$, Stevan Ramirez ${ }^{3}$, Joni Steinman $^{4}, \mathrm{Al} \mathrm{Kinel}{ }^{4}$, Shari Kinel ${ }^{3}$

${ }^{1}$ Georgetown University, Washington, DC, USA

${ }^{2}$ Griffin Foundation, Potomac, MD, USA

${ }^{3}$ Huntington Study Group, Rochester, NY, USA

${ }^{4}$ Strategic Interests, LLC, Rochester, NY, USA

\section{Abstract}

Background: There is little known about the quality of Huntington disease (HD) care in the USA. Improved care 
can reduce disease burden for patients and their family members.

Objectives: To measure satisfaction with HD care in the USA, and to identify gaps in HD care.

Methods: Surveys were developed in collaboration with PriceWaterhouseCoopers and Huntington Study Group (HSG) family focus groups, and disseminated via patient advocacy/research participant social media during 6 weeks in spring, 2017.

Results: Total valid responses numbered 856, including 626 caregiver respondents and 230 patient responses. Respondents received care from Huntington's Disease Society of America Centers of Excellence; HSG; and other HD specialty centers, primary care providers, movement disorder clinics, and other settings. Only $30 \%$ of respondents reported receiving HD specialty care. Patients and caregivers reported searching online ( $81 \%$ of respondents in the last 12 months) for information about HD, researching clinical trials (61\% in last 12 months), looking up at-home care-giving tips (45\% in last 12 months), and using HD social media channels (62\% in last 12 months). Patients reported the highest quality of care associated (from highest to lowest) with HD specialists, speech pathologists, and occupational therapists. Caregivers found HD specialists, neurologists, psychiatrists/psychologists, and genetic counselors provided the highest quality of care (from highest to lowest). Informal care needs were generally not being met. Patients and caregivers emphasized a need for support in financial planning and accessing care. They also expressed a need for more HD education in the medical community.

Conclusions: There is need for more support for patients with HD and their families. People desire more credible, accessible information. Improving online resources can help fill information gaps. Increased HD specialty training is associated with higher satisfaction with care. Improving resources available to patients and families should be a goal for HD organizations, along with measurement of patient outcomes.

\section{Poster \#3}

Measurement of Huntington's Irritability and Aggression in the Azevan Pharmaceuticals-NeuroNext STAIR Study Karen Anderson ${ }^{1}$, Steve Hersch ${ }^{2,3}$, Jeffrey Long ${ }^{4}$, Merit Cudkowicz ${ }^{3}$, Christopher Coffey ${ }^{5}$, Jon Yankey ${ }^{5}$, Michele Costigan $^{5}$, Sabrina Raulerson ${ }^{3}$, Samantha Berg ${ }^{3}$, Robin Conwit $^{6}$, Codrin Lungu ${ }^{6}$, Michael Brownstein ${ }^{7}$, Neal Simon ${ }^{7}$ ${ }^{1}$ Georgetown University School of Medicine, Washington, DC, USA

${ }^{2}$ Voyager Therapeutics Inc., Cambridge, MA, USA

${ }^{3}$ Massachusetts General Hospital, Boston, MA, USA

${ }^{4}$ Department of Psychiatry and Department of Biostatistics, University of Iowa, IA, USA

${ }^{5}$ Department of Biostatistics, University of Iowa, Iowa City, IA, USA
${ }^{6}$ National Institute of Neurological Disorders and Stroke, Bethesda, MD, USA

${ }^{7}$ Azevan Pharmaceuticals, Inc., Bethlehem, PA, USA

\section{Abstract}

Background: Irritability and aggression are common, challenging problems in Huntington's disease (HD), and there are no approved treatments. Current options rely on drugs developed for other indications and may lead to polypharmacy, inadequate treatment response, and undesirable side effects. Better characterization of these symptoms is needed to develop new treatments.

Objectives: To characterize the severity of irritability and aggression in patients with HD in the ongoing Azevan Pharmaceuticals-NeuroNext STAIR study (NCT02507284) of a vasopressin antagonist (SRX246) using the Irritability Scale (IS), the only scale developed specifically for HD, and the Cohen-Mansfield Agitation Inventory (CMAI), designed to capture problematic behaviors in dementia. This is a clinical trial of a new medication that may help to treat irritability and aggression. Methods: We are looking at how well various patient and caregiver scales work to measure irritability and aggression in this ongoing study. To provide a population enriched for irritability and aggression, the Unified HD Rating Scale Behavioral Irritability and Aggression items were used as a screening tool for study entry. The IS is a 14-item scale, with scoring ranging from 0 to 56. Higher scores indicate more severe symptomatology. The CMAI is a 29 -item study partner report.

Results: Sixty-nine patients were enrolled in the study as of 31 July 2017 . Thirty-five $(50.7 \%)$ were female. At baseline, mean \pm SD IS score for patients with HD was $22.5 \pm 5.3$ and $32.5 \pm 7.3$ for study partner reports on patients. CMAI scores indicated Physical/Aggressive Behavior in 16 patients (23.2\%), Physical/Non-Aggressive Behavior in 18 (26.1\%), and Verbal/Aggressive Behavior in 35 (50.7\%). CMAI groups were not mutually exclusive.

Conclusions: In a population of patients with HD with irritability, the IS and CMAI were useful for measurement of these symptoms. Study partners reported higher levels of irritability in patients with HD than did the patients themselves. This study demonstrates that irritability and aggression can be measured in patients with HD using self and study partner reports. Future work will determine whether these scales reliably assess behavioral changes in clinical trials with patients with HD.

\section{Poster \#4 Award Winner for Kimberly Quaid Genetics Abstract}

Prenatal Genetic Testing for Huntington's Disease: Ethical Considerations

Setareh Ashtiani ${ }^{1}$, Jasmine Doonanco ${ }^{2}$, Oksana Suchowersky ${ }^{1,2}$ 
${ }^{1}$ Department of Medicine (Neurology), University of Alberta, Canada

${ }^{2}$ Department of Medical Genetics, University of Alberta, Canada

\begin{abstract}
Background: The predictive genetic testing protocol for Huntington's disease (HD) is well established and is offered to individuals at $50 \%$ or $25 \%$ risk for the condition. The international guidelines for predictive testing are followed. Direct prenatal testing is offered to couples when the fetus is at 50\% risk, after extensive counseling and with the intention of termination of an affected pregnancy.
\end{abstract}

Case description: We present the case of a woman of childbearing age who asked us to test her fetus for the HD-causing expansion, shortly after completing the predictive testing process, during which she learned she carried the mutation. Extensive counseling identified that the patient's desire to know the information was solely for planning future pregnancies, and that the pregnancy would be continued regardless of the gene status of the fetus. As this was contrary to our current guidelines, opinions were obtained from the hospital ethicist and other HD clinicians/geneticists. Ethical considerations included that a mutation-positive result can harm more than help a child, through potential impacts such as alteration of selfimage and psychological distress. Other considerations include the right of the future child to decide whether or not to undergo presymptomatic test in adulthood. The principle of respect for autonomy dictates that individuals make these decisions for themselves. Thus, some at-risk asymptomatic adults may decide to have predictive testing for $\mathrm{HD}$, whereas research and our team's clinical experience suggest that most at-risk individuals decide against predictive genetic testing. Following significant discussions and ethical considerations, the decision was that in spite of the patient's wishes, prenatal testing could not be offered as it would be the same as testing an asymptomatic child for HD.

\section{Poster \#5}

Special Considerations in Cases with an Absence of Documented Family History of Huntington's Disease

Tanya Bardakjian, Kaylee Naczi, Rachel Paul, Pedro Gonzalez-Alegre

University of Pennsylvania, Philadelphia, PA, USA

\section{Abstract}

Background: Huntington's disease (HD) is an autosomal dominant condition. Accurate diagnosis of HD is critical for appropriate clinical management and genetic counseling. The absence of a documented or known family history of HD is often misleading to nonexperienced clinicians, who should be cognizant of special counseling considerations in these cases. Objectives: To describe the diagnostic challenges and genetic counseling considerations in individuals diagnosed with HD without a documented family history.

Methods: We identified all patients seen in the HD clinic in a 2-year period (July 2015-July 2017). Charts were reviewed and those without a diagnosis of HD excluded. For the remainder of the patients (HD), we reviewed the chart to determine if there was a known family history of HD at the time of diagnosis. We included those who were the first with a HD diagnosis in the family.

Results: A total of 176 patients were seen at the HD Center in the study period; 129 had a diagnosis of HD. Of these, 32 $(25 \%)$ were the-first known diagnosis in the family. Of those, 10 had evidence, in retrospect, that a family member likely had HD; 7 were adopted; 5 had no contact with the parent or family member affected by HD; 9 had an entirely negative history; and there was 1 case of nonpaternity. Subjective analysis of the response to the diagnosis for those without and with a family history will be presented.

Conclusions: We have a high rate of first HD diagnosis likely due to referral bias. Obtaining a detailed 3-generation pedigree of an individual presenting with symptoms suggestive of HD, without a known family history, often reveals family members with motor, cognitive, or psychiatric symptoms. Reaction to diagnosis differs between those with and without a known HD family history.

\section{Poster \#6 \\ Understanding the Educational Needs of Neurologists Who Manage Patients With Huntington's Disease: A Study Analyzing Both Clinician and Patient Perspectives Justin Barnes, Lee Whitworth, Brandon Coleman, Sharon Hwang, Gregory Salinas \\ CE Outcomes, Birmingham, AL, USA}

\section{Abstract}

Background: Huntington's disease (HD) has a complex presentation that varies widely between patients and poses a significant challenge to neurologists. Continuing education (CE) programs offer an effective means of improving physician performance, but to be most effective, CE must target the greatest areas of need. There are few data available on neurologist HD practice patterns or the perspectives of patients with HD.

Methods: Practice patterns of U.S. general neurologists, and neurologists practicing in Huntington's Disease Society of America (HDSA) Centers of Excellence (CoE), were assessed using an online case vignette survey. Case vignettes are an established, validated method for assessing physician practice patterns. The survey included 2 patient cases: a 51-year-old 
man with progressive motor difficulties and mild chorea; and a 47-year-old woman with severe depression, suicidal ideation, and moderate chorea. Practice patterns were compared with HDSA guidelines to identify areas that may benefit from CE. An additional survey was developed to investigate perceptions and educational needs of patients with HD/their caregivers.

Results: Responses were collected from 94 general neurologists, $33 \mathrm{CoE}$ neurologists, 30 patients, and 69 caregivers. Opportunities for neurologist $\mathrm{CE}$ were identified with regard to use of nonpharmacologic management strategies in earlystage patients; use of tetrabenazine in patients with a history of depression and suicidal ideation; and identification of alternative therapies for tetrabenazine. The patient/caregiver study identified differing treatment priorities between patients and caregivers. Patients/caregivers seen by movement disorder specialists had more confidence in their physician than those seen by general neurologists.

Conclusions: This study identified several key aspects of the care of patients with HD that may benefit from future $\mathrm{CE}$ programming. Education targeting these aspects of management will address the greatest areas of need and enable neurologists to provide optimal care to patients with HD.

\section{Poster \#7}

Safety and Tolerability of SRX246 in Irritable/Aggressive Subjects with Huntington's Disease (STAIR): A Phase II Exploratory Clinical Trial

Sabrina Raulerson ${ }^{1}$, Samantha Berg ${ }^{1}$, Michele Costigan ${ }^{2}$, Steve Hersch ${ }^{3}$, Karen Anderson ${ }^{4}$, Jeffrey Long ${ }^{2}$, Chris Coffey $^{2}$, Jon Yankey ${ }^{2}$, Merit Cudkowicz ${ }^{1}$, Robin Conwit ${ }^{5}$, Codrin Lungu ${ }^{5}$, Michael Brownstein ${ }^{6}$, Neal Simon ${ }^{6}$

${ }^{1}$ Massachusetts General Hospital, Boston, MA, USA

${ }^{2}$ Biostatistics, University of Iowa, Iowa City, IA, USA

${ }^{3}$ Voyager Therapeutics Inc., Cambridge, MA, and Massachusetts General Hospital/Harvard Medical School, Charlestown, MA, USA

${ }^{4}$ Georgetown University School of Medicine, Washington, DC, USA

${ }^{5}$ National Institute of Neurological Disorders and Stroke, Bethesda, MD, USA

${ }^{6}$ Azevan Pharmaceuticals, Inc., Bethlehem, PA, USA

\section{Abstract}

Background: Psychiatric symptoms, including irritability and aggression, are common in patients with Huntington's disease (HD). These are highly distressing aspects of the disease; they adversely impact daily life and often result in institutionalization. Effective treatments are lacking, and wellvalidated scales for measuring changes in these symptoms are not available. The STAIR Phase II clinical trial in patients with HD ( $n=108$; NCT02507284) rigorously evaluates the tolerability of a new drug, SRX246; provides additional safety data on the compound; and explores various rating scales for the assessment of changes in irritability and aggression. The objective is to obtain data that can be used to plan future Phase IIb or III clinical trials.

Methods: STAIR is a 3-arm, multicenter, randomized, placebo-controlled, double-blind, 12-week dose escalation study sponsored by Azevan Pharmaceuticals and coordinated by the National Institute of Neurological Disorders and Stroke (NINDS) NeuroNEXT network (22 sites). Subjects are randomized to receive placebo, or escalate from $80 \mathrm{mg}$ ( 2 weeks) to $120 \mathrm{mg}$, up to a maximum dose $160 \mathrm{mg}$ twice daily of SRX246 for 12 weeks. Each subject has a study partner to assist with visits, taking study medication, and providing feedback about the subject's mood and behavior. As of 7 August 2017, 70 subjects have been randomized and 44 have completed the protocol. Compliance has been very good, and while we are blinded, the adverse event profile and tolerability are consistent with other trials that show good safety and tolerability.

Results: The test compound, SRX246, is a first-in-class, orally bioavailable, brain-penetrant vasopressin 1a receptor antagonist. Preclinical pharmacology and an experimental medicine functional magnetic resonance imaging study in healthy volunteers showed that SRX246 has central nervous system effects after oral administration and that it modulates brain circuits involved in responses to stimuli that elicit aggression/ fear. These findings strongly suggested that SRX246 might have a beneficial effect on the irritability and aggression seen in a sizable proportion of patients with HD.

Financial support and disclosure: Funding for the project is provided by Azevan Pharmaceuticals, Inc., and NINDS grants 1 U44 NS090616 [M.J. Brownstein, principal investigator (PI)], UO1 NS077179 (M. Cudkowciz, PI), and U01 NS077352 (C. Coffey, PI). N.G. Simon and M.J. Brownstein hold equity in Azevan Pharmaceuticals and are officers in the company. Karen Anderson is a paid consultant to Azevan.

\section{Poster \#8}

Neurofilament Light Chain in Blood as a Predictor of Onset, Progression, and Brain Atrophy in Huntington's Disease: A Longitudinal Cohort Analysis

Lauren M. Byrne ${ }^{1}$, Filipe B. Rodrigues ${ }^{1}$, Kaj Blennow ${ }^{2,3}$, Alexandra Durr ${ }^{4}$, Blair R. Leavitt ${ }^{5}$, Raymund A.C. Roos ${ }^{6}$, Rachael I. Scahill ${ }^{1}$, Sarah J. Tabrizi ${ }^{1}$, Henrik Zetterberg ${ }^{1,2,3}$, Douglas Langbehn ${ }^{7}$, Edward J. Wild ${ }^{1}$

${ }^{1}$ UCL Institute of Neurology, London, UK

${ }^{2}$ Department of Psychiatry and Neurochemistry, Institute of Neuroscience and Physiology, the Sahlgrenska Academy at the University of Gothenburg, MöIndal, Sweden 
${ }^{3}$ Clinical Neurochemistry Laboratory, Sahlgrenska University Hospital, MöIndal, Sweden

${ }^{4}$ Institut du Cerveau et de la Moelle Épinière (ICM), Sorbonne Université, Paris, France

${ }^{5}$ Centre for Molecular Medicine and Therapeutics, University of British Columbia, Vancouver, BC, Canada

${ }^{6}$ Leiden University, Leiden, The Netherlands

${ }^{7}$ University of Iowa, Iowa City, IA, USA

\section{Poster \#9}

Enroll-HD: A Clinical Research Platform for Huntington's Disease

Selene Capodarca ${ }^{1}$, Olivia Handley ${ }^{1}$, Jamie Levey ${ }^{1,2}$, Tim McLean $^{1}$, Bernhard Landwehrmeyer ${ }^{3}$, Cristina Sampaio ${ }^{2}$ (on behalf of the Enroll-HD Steering Committee)

${ }^{1}$ EHDN, Ulm University, Ulm, Germany

${ }^{2}$ CHDI Foundation, Princeton, NJ, USA

${ }^{3}$ Ulm University, Ulm, Germany

\begin{abstract}
Background: Blood biomarkers of neuronal damage could facilitate clinical management of and therapeutic development for Huntington's disease (HD). We investigated whether neurofilament light protein (NfL) in blood is a potential prognostic marker of neurodegeneration in patients with HD.

Methods: In the 3-year, 298-participant TRACK-HD cohort, we did a retrospective analysis of the relationship between plasma NfL and clinical and neuroimaging measures previously identified as being the strongest predictors of HD progression. Cross-sectional and longitudinal relationships were analyzed using random-effect models of within-subject correlation. In a separate 37-participant cohort, we quantified NfL in cerebrospinal fluid (CSF) and plasma.
\end{abstract}

Results: Mean concentrations of NfL in plasma at baseline were significantly higher in HTT mutation carriers than in controls $(3.63 \pm 0.54 \mathrm{log} \mathrm{pg} / \mathrm{ml}$ vs 2.68 $\pm 0.52 \log \mathrm{pg} / \mathrm{ml} ; p<0.0001)$ and the difference increased with disease stage. At any given time point, plasma NfL correlated with clinical and magnetic resonance imaging findings. In longitudinal analyses, baseline plasma NfL also correlated significantly with subsequent decline in cognition (Symbol Digit Modalities Test $\mathrm{r}=0.374, p<0.0001$; Stroop Word Reading (SWR) ( $\mathrm{r}=0.248, p=0.0033)$, Total Functional Capacity (TFC) $(\mathrm{r}=0.289, p=0.0264)$, and brain atrophy (caudate $\mathrm{r}=0.178, p=0.0087$; whole brain $\mathrm{r}=0.602, p<0.0001$; gray matter $\mathrm{r}=0.518, p<$ 0.0001 ; white matter $\mathrm{r}=0.588, p<0.0001$; ventricular expansion $r=0.589, p<0.0001)$. All changes, except SWR and TFC, remained significant after adjustment for age and $\mathrm{CAG}$ repeat count. In premanifest individuals with HD, plasma NfL at baseline was associated with subsequent clinical onset during the 3-year follow-up period (hazard ratio 3.29 per $\log \mathrm{pg} / \mathrm{ml}$, 95\% confidence interval $1.48-7.34 ; p=0.0036)$. Concentrations of $\mathrm{NfL}$ in CSF and plasma were correlated in mutation carriers $(\mathrm{r}$ $=0.868, p<0.0001)$.

Conclusions: NfL in plasma shows promise as a potential prognostic blood biomarker of disease onset and progression in HD.

\begin{abstract}
Background: Enroll-HD is a clinical research platform that includes an observational, prospective study of Huntington's disease (HD).

Objectives: To provide an overview of the platform activities supported by Enroll-HD.

Methods: Enroll-HD is a global research platform for HD. At its core is a longitudinal observational study on HD. Clinical data and biosamples on an HD population are collected annually and undergo a rigorous monitoring process.
\end{abstract}

Results: The study comprises 14,457 participants, 143 sites, and 15 countries. Forty-five percent are HD stages 1 to $2 ; 18 \%$ are premanifest HD. The study aims to increase the percentage of premanifest/early-stage participants to support clinical trials. The current data set includes data on 8714 participants. More than 150 data set requests and 12 biosample distributions have been approved. Platform Services expedites the start-up and conduct of HD studies/clinical trials. Two studies (HDClarity and FuRST2.0) have utilized platform services; for example, site selection, feasibility, participant eligibility, and monitoring, with $\geq 5$ studies planned for 2017-18. Potentially eligible participants for clinical trials can be identified from the database. In total, 1011 participants were randomized in 3 clinical trials from approximately 9000 manifest Enroll-HD participants (70 Enroll-HD sites). The Clinical Training Platform, the product of close collaboration between European Huntington's Disease Network, Cure Huntington's Disease Initiative (CHDI), and Huntington Study Group, was launched in 2017. It enables faster, more cost-effective startup of studies/clinical trials, standardizes the quality of training, and reduces workload at sites.

Conclusions: The Enroll-HD platform continues to accelerate the development of new therapeutics for HD, to inform our understanding of the disease, and to lead to improvements in care. The Enroll-HD study provides high-quality data and biosamples to researchers.

Acknowledgements: Enroll-HD is sponsored by CHDI Foundation, a nonprofit organization dedicated to developing therapeutics for HD. Enroll-HD would not be possible without the vital contribution of the research participants and their families 


\section{Poster \#10}

Effects of a Physical Therapy Home Program Plus Community-Based Exercise Classes for A Person with Early-Stage Huntington's Disease: A Case Report

Adrianna Carey

Good Shepherd Penn Partners/Penn Therapy \& Fitness, HDSA Center of Excellence, University of Pennsylvania, USA

\section{Abstract}

Background: Huntington's disease (HD) is a progressive, neurodegenerative disease characterized by symptomology in motor, psychiatric, and cognitive realms. Those with early-stage HD typically demonstrate minimal motor symptoms that may not interfere with a person's functional ability and may prohibit need for skilled intervention via physical therapy (PT) in the outpatient setting. This case study outlines changes in standardized outcome measures from initial evaluation to annual follow-up at a Huntington's Disease Society of America Center of Excellence (CoE) after practice of a PT-prescribed home program plus community-based exercise classes.

Case description: The patient was a 52-year-old woman with a diagnosis of HD referred to outpatient PT by a movement disorder specialist and evaluated as part of the CoE HD clinic at the University of Pennsylvania.

Outcomes: Primary outcomes included Functional Gait Assessment (FGA) and self-reported falls and were assessed at initial evaluation and at 14-month follow-up. Intervention consisted of functional training, postural stability exercise, and balance activities to fulfill the patient's goal of modifying her current exercise routine to focus on anticipated motor symptoms of HD. A total of 3 visits in 14 months were completed. On initial evaluation, the patient scored $27 / 30$ on the FGA, indicating a low risk for falls. Despite practice of a PT home exercise program, plus community-based exercise classes 5 times weekly, a decline in FGA testing was observed, with the patient scoring 21/30 at follow-up, indicating increased risk for falls. Changes in FGA testing were observed in gait with vertical head turns, gait with narrow base of support, and gait with eyes-closed conditions. The patient reported no falls during the 14 months between initial evaluation and follow-up.

Conclusions: This case study demonstrates that use of a home exercise program with community-based exercise classes in a person with early-stage HD is a feasible option. Despite decline on the FGA, the patient reported no falls from initial evaluation to follow-up.

\section{Poster \#11}

Exploring the Transcriptional Consequences of LongTerm Huntingtin Lowering in vivo

Sydney R. Coffey ${ }^{1}$, Jeffrey P. Cantle ${ }^{1}$, Robert M. Bragg ${ }^{1}$, Samuel R.W. Legg ${ }^{1}$, Fiona Kinsella ${ }^{1}$, Kylee Haagenson ${ }^{1}$,
Will Hovander ${ }^{1}$, Miguel Sena-Esteves ${ }^{2}$, David S. Howland ${ }^{3}$, Jeffrey B. Carroll ${ }^{1}$

${ }^{1}$ Behavioral Neuroscience Program, Department of Psychology, Western Washington University, Bellingham, WA, USA

${ }^{2}$ Department of Neurology, Horae Gene Therapy Center, University of Massachusetts Medical School, Worcester, MA, USA

${ }^{3}$ CHDI Foundation, Princeton, NJ, USA

\section{Abstract}

Background: We are interested in the long-term consequences of huntingtin lowering. Global Htt knockout is embryonic lethal, and very few tissue-specific huntingtin (Htt) knockouts have been described, which limits our understanding of Htt loss-offunction liabilities in the context of a wide range of cell types. Methods: We are exploring the impact of Htt loss of function in the liver using a 2-part strategy of hepatocyte-specific knockout mice (HttLKO) and mice treated with virally delivered zinc finger repressor proteins (ZFPs). This approach enables comparison of developmental Htt loss-of-function phenotypes in HttLKO mice, which completely lack postnatal Htt expression, and acute Htt loss-of-function phenotypes in adult mice treated with ZFPs. At 11 weeks of age, $\mathrm{HttQ} 111 /^{+}$and $\mathrm{Htt}^{+/+}$mice were intravenously injected with self-complementary adeno-associated virus (serotype 9) containing ZFP constructs targeting panHtt, mHtt, or a control ZFP lacking the DNA binding domain. Motivated by the hypothesis that Htt loss-of-function liabilities may only reveal themselves in the context of metabolic stressors, we conducted a series of metabolic challenges beginning at 23 weeks of age, 12 weeks after injection.

Results: No differences were observed in glucose metabolism following glucose, pyruvate, or insulin tolerance tests, nor were differences observed in xenobiotic clearance of midazolam or zoxazolamine, as measured by loss of righting reflex. By 25 weeks of age, HttLKO mice weighed approximately $10 \%$ less than their wild type and HttQ111/ ${ }^{+}$counterparts, regardless of treatment, but this difference was not caused by liver atrophy. We have generated RNASeq profiles of livers treated with ZFPs from HttLKO/LKO mice, the results of which will be discussed.

\section{Poster \#12}

FightHD Neuroplasticity-Based Treatment for Huntington's Disease

Melanie Cheung ${ }^{1}$, Leo Sugrue ${ }^{2}$, Elizabeth Tong ${ }^{2}$, Jo Dysart ${ }^{3}$, Greg Finucane $^{4}$, Lynette Tippett ${ }^{5}$, Margaret Dudley ${ }^{5}$, Soweeta Fort-D'Ath $^{1}$, Richard Faull ${ }^{1}$, Mike Dragunow ${ }^{6}$, Mike Merzenich $^{7}$

${ }^{1}$ Department of Anatomy and Medical Imaging, University of Auckland, Auckland, NZ 
${ }^{2}$ Department of Radiology and Biomedical Imaging, University of California San Francisco, San Francisco, CA, USA

${ }^{3}$ Liaison Psychiatry, Auckland District Health Board, Auckland, NZ

${ }^{4}$ Liaison Psychiatry, Waitemata District Health Board, Auckland, NZ

${ }^{5}$ School of Psychology, University of Auckland, Auckland, NZ

${ }^{6}$ Department of Pharmacology, University of Auckland, Auckland, NZ

${ }^{7}$ Posit Science, San Francisco, CA, USA

\begin{abstract}
Background: We have developed FightHD, a novel neuroplasticity-based cognitive training program to address early cognitive changes in Huntington's disease (HD; attention, speed of processing, working memory, emotional recognition, decision-making, switching, sequencing, impulse control, language, auditory perception, and psychomotor control). The treatment harnesses neuroplasticity (the brain's natural ability to change in response to the input it receives) to fight HD progression. It is thought that brain cells begin to die when they lose their connections to other brain cells. We are testing whether stopping the cells from becoming disconnected can slow down the brain cell death that causes HD. FightHD strengthens brain processes that are damaged early in HD.
\end{abstract}

Results: Our preliminary results show for the first time that $100 \mathrm{~h}$ of FightHD neuroplasticity-based cognitive training is driving positive neurological changes in brain networks subserving 7 major domains affected by HD (visual, sensorimotor, attention, amygdala, frontoparietal, default mode, and basal ganglia) as measured by restingstate functional magnetic resonance imaging. Specifically, we observe increased functional connectivity both within and between networks following FightHD neuroplasticitybased cognitive training in 7 presymptomatic HD (preHD) participants compared with 5 patients with pre-HD that did active-control training. Synaptic and dendritic disconnection are key components of neurodegeneration, raising the possibility that the training-based changes in functional connectivity we observe may be a marker of slowed clinical disease progression. Concurrently, brain-derived neurotrophic factor (BDNF) was elevated in 8/12 neuroplasticity-trained participants with pre-HD (average increase of $1366 \mathrm{pg}$ ) versus $4 / 8$ active control-trained participants with pre-HD. Note that although half the active control-trained patients with pre-HDs had increased BDNF, overall there was still an average decrease of $687 \mathrm{pg}$ in this group. Since BDNF plays a role in neuronal survival, growth, and differentiation of new neurons and synapses, elevation of BDNF alone warrants further investigation of FightHD neuroplasticity training as a treatment for HD.
Conclusions: We have learned that people with HD who "brain train" for $100 \mathrm{~h}$ on FightHD have increased connections in some crucial brain regions where the cells usually die (vs people with HD who brain train for $100 \mathrm{~h}$ on control exercises that have no neuroplasticity component). We see increased connections in the regions responsible for movement, coordination, visual processing, concentration, mental flexibility, and emotional processing in their magnetic resonance imaging scans. We are still trying to understand if this means that we have been able to slow disease progression. We may not know the answer to this until our 5-year follow-up assessments with patients with HD who have trained on FightHD, but it does seem hopeful. We also see that BDNF increases in most people who brain train with FightHD. Some scientists think that BDNF should be given to people with HD as a drug because it will help their brains to stay healthy. Since FightHD brain plasticity-based training gets the brain to produce more BDNF than is usually seen in HD, FightHD is potentially protective against brain cells from dying. One of the most promising things about FightHD brain plasticitybased training is that our patients with HD love it. Many of them report having more energy, sharper thinking, and a sense of hope (for the first time in a long time). This is, therefore, very exciting.

\section{Poster \#13}

Review of Neuropsychiatry in a Huntington's Disease Clinic

Elvina May-Yin $\mathrm{Chu}^{1,2}$, Caitlin Hartney ${ }^{3}$

${ }^{I}$ The National Hospital for Neurology and Neurosurgery, UCLH NHS Foundation Trust, London, UK

${ }^{2}$ St Andrew's Healthcare, Northampton, UK

${ }^{3}$ Kings College London, London, UK

\begin{abstract}
Background: Huntington's disease (HD) is primarily treated as a neurological condition. Despite a high prevalence of psychiatric comorbidity, few studies have investigated psychiatric treatment within an HD outpatient clinic setting. The National Hospital for Neurology and Neurosurgery has one of the largest tertiary referral centers for HD in the UK, and clinical provision includes input from a consultant neuropsychiatrist on a case-bycase referral basis.

Methods: A retrospective case-note review of outpatients with a confirmed diagnosis of HD attending the clinic at The National Hospital for Neurology and Neurosurgery between March and June 2016 was carried out. Patients were grouped under care of a neurologist only, or under joint care of a neurologist and neuropsychiatrist. Characteristics and prevalence of psychiatric symptoms and diagnoses were compared
\end{abstract}


between groups, in addition to psychotropic medications prescribed and referral rates to Community Mental Health Teams (CMHTs). Multivariate analysis was utilized to determine if there was a difference in symptom outcome and whether there was a change in psychotropic medication prescribing following neuropsychiatry review.

Results: This study found a high prevalence of psychiatric symptoms (70\%) in both groups, with apathy and depression most commonly reported. Remission or symptom resolution was reported in $43 \%$ of patients attending follow-up. Both groups received increased psychotropic prescribing during follow-up, with the neuropsychiatry patients more likely to be prescribed additional psychotropics and referred for psychotherapy and CMHT input.

Conclusions: Both patient groups reported decreases in symptoms at follow-up, with neuropsychiatry patients receiving more intensive psychiatric assessment and treatment. Considering that psychiatric symptoms and history were more prominent in this group, the study demonstrates both clinical need and utility for neuropsychiatry within the HD outpatient setting

\section{Poster \#14}

Investigating the Clinical Predictors of Depression in Huntington's Disease: An Enroll-HD Database Study

Gabriela Delevati Colpo, Natalia Pessoa Rocha, Erin FurrStimming, Antonio Teixeira

University of Texas Health Science Center at Houston, Houston, TX, USA

\footnotetext{
Abstract

Background: Besides motor symptoms, Huntington's disease (HD) is marked by cognitive impairment and behavioral changes. Among several psychiatric symptoms, depression is of great relevance as it is considered a significant burden for individuals with HD and their caregivers. In addition, the presence of depression is a predictor of suicidal ideations, attempts, and completion. Despite the high frequency and significance of depressive symptoms in HD, there is a lack of predictive and prognostic data to guide clinicians in its management. This study was designed to investigate clinical predictors of depression in patients with HD.

Methods: This study included 2303 subjects with manifest HD from the Enroll-HD Database (December 2015). A binary logistic regression (backward stepwise approach) was performed to ascertain the effects of general clinical characteristics, medical history of substance use and psychiatric/ behavioral problems, motor and functional capacity, and cognitive performance on the likelihood that patients with HD present depression or suicidal ideation.
}

Results: In the final model $\left(\chi^{2}=270.648, p<0.0001\right)$, younger age at HD clinical diagnosis [odds ratio (OR) 0.969, $p=$ 0.001 ], lower number of CAG repeats (OR 0.904, $p=0.002$ ), clinical history of irritability (OR $2.119, p<0.0001$ ), violent/ aggressive behavior (OR 1.492, $p=0.040$ ), apathy (OR 3.631, $p<0.0001$ ), psychosis (OR 3.658, $p=0.001$ ) and cognitive impairment (OR 1.409, $p=0.024$ )], and female sex (OR $2.124, p<0.0001)$ remained as predictors on the likelihood of depression. Additional analyses revealed that depression was significantly associated with suicidal ideation $(52.2 \%$ of patients with HD presenting history of depression had suicidal ideation, whereas only $7.5 \%$ of patients without history of depression had suicidal ideation; $p<0.0001$ ).

Conclusions: Depression is associated with a younger age at motor manifestations and a lower number of CAG repeats. Female sex, behavioral problems (noteworthy apathy and psychosis), and poorer cognitive performance were also predictors for depression in HD. This study helps to identify and characterize depression in individuals with HD and to treat the patients with more effectiveness, improving their quality of life and the quality of life of their caregivers.

\section{Poster \#15 \\ Optimization of Chemically Modified siRNA for Allele- Specific Targeting of Human Huntingtin}

Faith Conroy, Julia Alterman, Matthew Hassler, Dimas Echeverria, Neil Aronin, Edith Pfister, Anastasia Khvorova University of Massachusetts Medical School, Worcester, MA, USA

\begin{abstract}
Background: Huntington's disease (HD) is a devastating autosomal dominant neurodegenerative disease caused by expansion of the CAG repeat region of the Huntington gene. Suppression of huntingtin (HTT) by RNA interference (RNAi) is a promising therapeutic strategy for treating the disease. Previously, we have described a fully chemically modified small interfering RNA (hsiRNA) scaffold that provides significant spread and long-term silencing after a single intracerebroventricular injection into the mouse brain. The major caveat of this hsiRNA is that silencing of HTT mRNA is nondiscriminate, and effects of total silencing of wild-type HTT are unknown.
\end{abstract}

Methods: Here, we describe a method for generating fully modified hsiRNAs with optimal allelic discrimination. For our initial screen, we chose to target single nucleotide polymorphism (SNP) site rs362273 in exon 57 of the Huntingtin gene, a site that is heterozygous in $\sim 30 \%$ of patients with HD. We screened a panel of hsiRNAs, placing the SNP at each position along the hsiRNA. After selecting the best SNP 
position, we performed a secondary screen, adding a single additional mismatch to the hsiRNA. We selected the hsiRNA with the greatest SNP discrimination and designed compounds with alternate backbone modifications to optimize efficacy and discrimination. We show that the additional mismatch in this chemical scaffold prevents silencing of the nontargeted allele, with minimal impact on silencing of the target allele in vitro. The pattern of the SNP and mismatch positions is sequence specific, but the same approach can be used to design hsiRNAs targeting other heterozygous SNPs in patients with HD.

Conclusions: hsiRNAs have allowed us to achieve full silencing of the huntingtin protein via RNAi; however, selective silencing of only the mutant allele has been difficult to perfect. We have established a method for optimizing allelic discrimination, utilizing hsiRNAs, with unique chemical modification patterns, which target a patient-specific heterozygous SNP in the HTT mRNA.

\section{Poster \#16}

Handwriting Movement Abnormalities as an Early Biomarker of Transition to Manifest Huntington's Disease Sungmee Park ${ }^{1}$, Jody Corey-Bloom ${ }^{1}$, Ajay Nathan ${ }^{1}$, Chase Snell $^{1}$, Michael Caligiuri ${ }^{2}$

${ }^{1}$ Department of Neurology, University of California, San Diego, La Jolla, CA, USA

${ }^{2}$ Department of Psychiatry, University of California, San Diego, La Jolla, CA, USA

\footnotetext{
Abstract

Background: Prior research has demonstrated that measures of handwriting kinematics are sensitive to mild preclinical motor abnormalities stemming from other basal ganglia disorders. The presence of a biomarker early in the transition period in HD may help efforts to pharmacologically manage or delay onset.

Objectives: To examine whether handwriting movement abnormalities are present prior to clinically manifest chorea in Huntington's disease (HD) and may serve as an early biomarker of transition to manifest disease.

Methods: Fifteen patients with premanifest HD (mean CAG 41.2), 15 patients with gene-negative HD (mean CAG 20.5), and 16 patients with manifest HD (mean CAG 42.7) completed handwriting tasks consisting of simple and complex loops, rapid circles, spirals, and a sentence, each repeated multiple times on a digitizing tablet using a noninking pen. Multiple measures of pen-stroke kinematics and pressure were extracted from these trials. The clinical status of each participant was characterized using the Unified Huntington's Disease Rating Scale (UHDRS) Total Motor Score (TMS) and Total Functional Capacity.
}

Results: Statistical comparisons revealed significant differences between patients with symptomatic HD and those with premanifest HD on several handwriting stroke features, particularly stroke duration and fluency. Discriminant function analyses were conducted to identify a multivariate model that distinguished premanifest from gene-negative subjects. An 8factor model consisting of stroke amplitude, velocity, fluency, and pressure (means and between-stroke variability) for complex loops distinguished premanifest from gene-negative subjects with $90 \%$ accuracy $[\mathrm{F}(8,21)=4.22 ; p<0.01]$. A discriminant function model based solely on UHDRS TMS score achieved only $63 \%$ accuracy for separating premanifest from gene-negative patients. We observed statistically significant positive correlations between $\mathrm{CAG}$ repeat number and several handwriting variables among premanifest and symptomatic patients with HD, including stroke duration and fluency for sentence writing and rapid circles.

Conclusions: These findings support the clinical utility of dynamic measures of handwriting kinematics as a potential early biomarker in HD.

\section{Poster \#17 Invited for Platform Presentation Salivary Biomarkers for Huntington's Disease} Jody Corey-Bloom ${ }^{1}$, Sungmee Park ${ }^{1}$, Ameera Haque ${ }^{1}$, Aeri Kim $^{1}$, Ajay Nathan ${ }^{1}$, Douglas Granger ${ }^{2}$, Steven Granger ${ }^{3}$, Elizabeth Thomas ${ }^{4}$

${ }^{1}$ Department of Neurosciences, University of California, San Diego, La Jolla, CA, USA

${ }^{2}$ Institute for Interdisciplinary Salivary Bioscience Research, University of California, Irvine, CA, USA

${ }^{3}$ Salimetrics, Carlsbad, CA, USA

${ }^{4}$ Department of Molecular and Cellular Neuroscience, The Scripps Research Institute, La Jolla, CA, USA

\begin{abstract}
Background: Peripheral biomarkers are greatly needed in Huntington's disease (HD) to anticipate onset of disease symptoms, monitor disease progression, and track potential therapeutic effects. Pathogenesis in HD is associated with expression of the mutant huntingtin $(\mathrm{mHtt})$ protein in the central nervous system; however, HD is also associated with abnormalities in peripheral tissues. Several therapeutic approaches directed at the production, processing, and/or turnover of $\mathrm{Htt}$ protein are under development for impending clinical trials. Measuring $\mathrm{Htt}$ and other disease proteins in peripheral cells represents an essential step in biomarker discovery for HD.

Objectives: To assess the potential for saliva to serve as an accessible biomarker for HD.

Methods: In the current study, we measured Htt protein in saliva from patients with manifest HD, gene-positive patients with premanifest HD, and age- and sex-matched normal
\end{abstract}


controls (NC; total $n=178)$ using Western blots and enzymelinked immunosorbent assay (ELISA) methods. Additional salivary analytes, including $\alpha$-amylase, cortisol, C-reactive protein (CRP), and uric acid, were also measured using standardized ELISAs.

Results: Salivary total Htt was significantly increased ( $p=$ 0.0012 ) in saliva from patients with HD (mean $0.775 \mathrm{ng} / \mathrm{ml}$ ) compared with $\mathrm{NC}$ (mean $0.359 \mathrm{ng} / \mathrm{ml}$ ). Salivary total $\mathrm{Htt}$ did not vary over time of day or over different days, nor were there age or sex effects. Additionally, salivary $\mathrm{mHtt}$ levels were higher in gene-positive patients with premanifest HD than in $\mathrm{NC}(p<0.05)$. CRP, a widely used biomarker of systemic inflammation, was found to be significantly $(p=0.025)$ elevated in premanifest HD $(9548 \mathrm{pg} / \mathrm{ml})$ compared with $\mathrm{NC}$ $(3399 \mathrm{pg} / \mathrm{ml}$ ) and may be an early marker for disease onset. Levels of other salivary proteins, $\alpha$-amylase and uric acid, were not significantly different between patients with HD, patients with premanifest patients, or NC.

Conclusions: Measurement of salivary $\mathrm{Htt}$ and other disease proteins offers significant promise as relevant, noninvasive biomarkers of disease onset and progression in HD. We found that it is not only possible to measure Htt protein in saliva, but also that other proteins that may be important indicators of disease onset and progression.

\section{Poster \#18}

The Impact of Living with Huntington's Disease on Family Caregivers and Those at Risk for the Disease

Amita Risbud ${ }^{1}$, Sungmee Park ${ }^{2}$, Ajay Nathan ${ }^{2}$, Jody CoreyBloom $^{2}$

${ }^{1}$ Rutgers Robert Wood Johnson Medical School, Piscataway, NJ, USA

${ }^{2}$ University of California, San Diego, La Jolla, CA, USA
AR-, 19 individuals who were at risk for HD but did not wish to know their gene status (GU), and 20 normal controls (NC). Results: Although NC and CGs were not statistically different with regard to cognitive, functional, and motoric measures, they showed significant differences with regard to behavior on the HD-BQ $(p<0.001)$ and HADS-SIS $(p=0.002)$. There were also significant differences among at-risk groups, in that $\mathrm{AR}+$ patients scored significantly higher on the HD-BQ $(p<$ $0.001)$, HADS-SIS $(p=0.006)$, and PBA-s $(p=0.003)$ compared with AR- patients. Likewise, GU patients, although unaware of their gene status, demonstrated significantly more behavioral symptoms and appeared qualitatively more like the gene-positive cohort.

Conclusions: CGs of patients with HD experience a number of behavioral issues, including depression and anxiety, which makes them less appropriate as $\mathrm{NC}$ for comparative studies. In addition, individuals at risk for HD who are gene-positive, or who do not know their genotype, appear to have significantly more behavioral symptomatology than those who are gene-negative. Our findings suggest that behavioral symptoms are greatest in individuals at-risk for and caring for patients with HD, and reinforce the need for appropriate social support, and perhaps intervention, in these vulnerable groups.

\section{Poster \#19}

Lumateperone (ITI-007): A Novel Approach for the Treatment of Multiple Neuropsychiatric and Neurologic Diseases

Sharon Mates, Kimberly E. Vanover, Eve Taylor, Jelena Saillard, Michal Weingart, Gretchen Snyder, Joseph P. Hendrick, Peng Li, Lawrence P. Wennogle, Robert E. Davis Intra-Cellular Therapies, Inc., New York, NY, USA 
dose range, lumateperone has been shown to restore natural sleep patterns in patients with primary insomnia and is currently being studied for the treatment of agitation associated with dementia, including Alzheimer's disease. As the dose is increased, additional pharmacologic activity is recruited, including interactions with dopamine receptors as a phosphoprotein modulator, which sees the compound acting as a presynaptic partial agonist and postsynaptic antagonist at dopamine D2 receptors, a dopamine D1 receptor-dependent indirect modulator of glutamate (both $N$-methyl-D-aspartate and $\alpha$-amino-3-hydroxy-5-methyl4-isoxazolepropionic acid), and as a serotonin reuptake inhibitor. In this higher dose range, lumateperone has been shown to be safe and effective in treating the symptoms associated with schizophrenia, including hallucinations and delusions, to improve comorbid depression, and to possess an overall response profile consistent with improved social function. Lumateperone is in phase III development for the treatment of agitation in patients with dementia (at low doses) and for the treatment of schizophrenia and bipolar depression (at higher doses). Lumateperone represents a potential new approach for the treatment of a broad array of psychiatric and neurologic symptoms, including symptoms associated with HD.

\section{Poster \#20}

Longitudinal Analysis of Mutant Allele-Specific Silencing in the YAC128xNSG Mouse Using Transcription Activator-Like Effectors

Peter Deng ${ }^{1}$, Julian Halmai ${ }^{2}$, Grace Tharmarajah ${ }^{3}$, Ivette Sandoval $^{4}$, Fredric Manfreddson ${ }^{4}$, David Segal ${ }^{1}$, Jan Nolta ${ }^{2}$, Kyle Fink ${ }^{5}$

${ }^{1}$ Genome Center, MIND Institute, and Biochemistry and Molecular Medicine, University of California, Davis, CA, USA

${ }^{2}$ Stem Cell Program and Institute for Regenerative Cures, University of California Davis Health Systems, Sacramento, CA, USA

${ }^{3}$ Precision Nanosystems Inc., Vancouver, BC, Canada

${ }^{4}$ Translational Science \& Molecular Medicine, Michigan State University, Grand Rapids, MI, USA

${ }^{5}$ Department of Neurology, University of California Davis Health Systems, Sacramento, CA, USA

\begin{abstract}
Background: Huntington's disease (HD) is an autosomal dominant neurodegenerative disorder characterized by the presence of a misfolded mutant huntingtin (muHTT) protein. Reduction of this protein via RNA interference has been extensively explored and has demonstrated attenuation of disease-related phenotypes. Our group has previously shown allele-specific silencing of the muHTT transcript in patient fibroblasts via transcriptional activator-like effectors (TALE)
\end{abstract}

specifically targeted to single nucleotide polymorphisms that are highly associated with the mutant allele. Furthermore, we have demonstrated a robust volume of distribution following unilateral striatal injection of our lipid nanoparticles (LNP)TALE across the ipsilateral striatum, subventricular zone, corpus collosum, and cerebral cortex, as well as presence of LNPTALE in the contralateral striatum and cortex. Molecular analysis of those regions demonstrated a significant $50 \%$ reduction of the muHTT and a trend toward reduction of the muHTT protein $48 \mathrm{~h}$ following injection.

Methods: Currently, our group is continuing to explore LNP, as well as adeno-associated virus (AAV), as putative delivery vehicles for our therapeutic TALE transgene in our novel NODSCID gamma YAC128 transgenic HD mouse model. Both delivery platforms have been explored in human clinical settings and offer multiple lenses in successfully delivering therapeutic transgenes in vivo. We have executed a longitudinal study evaluating an optimized LNP-TALE formulation and AAV9-TALE for volume of distribution, duration of transgene expression, duration of mutant allele silencing, and attenuation of HDrelated neuronal and behavioral pathologies.

Conclusions: Identification of a potent, widespread delivery vehicle and assessment of the long-term duration of expression and effect of our therapeutic transgene will be vital in the evaluation of our TALE as a viable therapeutic for HD.

\section{Poster \#21}

Latent Toxoplasma gondii Infection Promotes Neurodegeneration and Increases Soluble Mutant Huntingtin Levels in a Mouse Model of Huntington Disease

David W. Donley ${ }^{1,2}$, Teal Jenkins ${ }^{3}$, Sonal Agrawal ${ }^{2}$, Marley J. Realing $^{4}$, Vanita Chopra ${ }^{5}$, Steven Hersch ${ }^{5}$, Jason G. Gigley ${ }^{6}$, Jonathan H. Fox ${ }^{1,2}$

${ }^{1}$ Graduate Neuroscience Program, University of Wyoming, Laramie, WY, USA

${ }^{2}$ Department of Veterinary Sciences, University of Wyoming, Laramie, WY, USA

${ }^{3}$ WWAMI, College of Health Sciences, University of Wyoming, WY, USA

${ }^{4}$ Department of Microbiology, University of Wyoming, Laramie, WY, USA

${ }^{5}$ Mass General Institute for Neurodegenerative Disease, Charlestown, MA, USA

${ }^{6}$ Department of Molecular Biology, University of Wyoming, Laramie, WY, USA

\section{Abstract}

Background: Huntington's disease (HD) is a fatal neurodegenerative disorder caused by a dominant CAG repeat expansion in the huntingtin gene that manifests with motor, 
cognitive, and psychiatric symptoms. The kynurenine pathway of tryptophan metabolism is a neuroinflammatory pathway implicated in HD pathogenesis. Activity of the first enzyme in this pathway, indoleamine-2,3-dioxygenase (IDO), is increased in HD mouse and human brain. IDO activation also occurs as part of the immune response to the prevalent neuroinvasive human pathogen, Toxoplasma gondii and is required for prolonged control of the infection. Therefore, latent infection activates an HD-associated pathway and may exacerbate disease. We previously reported that N171-82Q HD mice have an altered response to acute $T$. gondii infection. However, the effect of latent $T$. gondii infection, characterized by intracellular parasite cysts and inflammation in brain, on HD progression is not known.

Methods: Here, we investigated the effects of latent $T$. gondii infection in YAC128 HD mice. Wild-type and HD mice were orally infected at 2 months of age with $T$. gondii or vehicle. Behavior was monitored longitudinally and mice were sacrificed at 12 months of age. Endpoint results, including IDO activity and brain iron measurements, will be reported.

Results: Interestingly, infection increased soluble mutant huntingtin levels in HD mice compared with HD mice without infection. In addition, brain weight, striatal volume, and number of striatal neurons of $T$. gondii-infected HD mice were significantly decreased compared with both non-infected HD mice and wild-type mice with infection. These results are consistent with HD potentiation by latent $T$. gondii infection.

\section{Poster \#22 Invited for Platform Presentation}

Clinical Development of VX15 Anti-Semaphorin 4D Antibody as a Potential Treatment for Huntington's Disease

Maurice Zauderer ${ }^{1}$, John Leonard ${ }^{1}$, Terrance Fisher ${ }^{1}$, E. Evans $^{1}$, D. Fisher ${ }^{1}$, L. Balch ${ }^{1}$, E. Smith ${ }^{1}$, A. Feigin ${ }^{2}$

${ }^{1}$ Vaccinex, Inc., Rochester, NY, USA

${ }^{2}$ For the Huntington Study Group SIGNAL investigators and coordinators, and the University of Rochester Clinical Trials Coordination Center, Rochester, NY, USA

\begin{abstract}
Background: Semaphorin 4D (SEMA4D) and its highaffinity receptor, Plexin B1 (PLXNB1), are expressed on brain neural, endothelial, and inflammatory cells. SEMA4D signaling via PLXNB1 triggers activation of inflammatory glial cells, inhibits migration and differentiation of oligodendrocyte precursor cells that can repair damage to myelin, and disrupts endothelial tight junctions that are required for the integrity of the blood-brain barrier (BBB). Chronic inflammation, neuronal degeneration, and disruption of the BBB are believed to play an important role in neuroinflammatory and neurodegenerative diseases. We have demonstrated in several
\end{abstract}

preclinical models, including HD transgenic mice, that antibody neutralization of SEMA4D ameliorates neurodegenerative processes.

Methods: The randomized, double-blind, phase II SIGNAL clinical trial is enrolling patients with prodromal and early-manifest HD treated with either VX15/2503 antiSEMA4D antibody or matching placebo. This study has an adaptive design and will evaluate safety, tolerability, and efficacy of monthly intravenous administration of the investigational agent as reflected in clinical features of HD, including cognition (HD Cognitive Assessment Battery) and quantitative motor assessment, as well as changes in volumetric magnetic resonance imaging (MRI) of defined brain regions and fludeoxyglucose positron emission tomography (FDG-PET) measures of glucose metabolism in brain.

Results: Cohort A enrolled 36 subjects and was completed in February 2017. The experience of the SIGNAL trial to date has shown that recruitment into this first study of a biologic administered intravenously to patients with late prodromal and early-manifest HD is feasible. No concerning safety signals were identified and participant retention and compliance with the study protocol has compared favorably with prior experience in other HD studies. We will report on biomarker analysis in cohort A of this study, with particular focus on MRI and FDG-PET imaging, and we will discuss how these data have informed the design of a larger cohort $\mathrm{B}$ in which active enrollment continues.

\section{Poster \#24}

A Multidisciplinary Huntington's Disease Clinic's Initial Experience with the New Canadian Legislation Allowing Medical Assistance in Dying

Clare A. Gibbons ${ }^{1,2}$, Wai Lun Alan Fung ${ }^{1,3}$, Blair Henry ${ }^{1,4}$, Sherali Esmail ${ }^{1,5}$

${ }^{1}$ North York General Hospital, North York, Ontario, Canada

${ }^{2}$ Department of Molecular Genetics, University of Toronto, Toronto, Canada

${ }^{3}$ Department of Psychiatry, University of Toronto, Toronto, Canada

${ }^{4}$ Department of Family and Community Medicine, University of Toronto, Toronto, Canada

${ }^{5}$ Department of Medicine, University of Toronto, Toronto, Canada

\section{Abstract}

Background: On 17 June 2016, Canada passed a law making medical assistance in dying (MAID) legal. To be eligible for MAID, the requestor must have a serious illness, be in an advanced state of decline that cannot be 
reversed, be experiencing unbearable physical or mental suffering that cannot be relieved, and natural death must be reasonably foreseeable. The requestor also needs the mental competency to be able to give informed consent, which requires an understanding of their medical diagnosis, available forms of treatments, and options available to relieve suffering. Under the current legislation, advanced directives are not permitted for MAID requests. Here, we outline 3 MAID requests received by the Multidisciplinary Huntington's Disease (HD) Clinic at North York General Hospital in the past year.

Methods: Case 1: A 34-year-old with moderate/severe motor symptoms and mild cognitive impairment. This individual experienced significant decline of activities of daily living over the past 2 years. All MAID requirements were met, and MAID was completed. Case 2: A 48-yearold with severe motor symptoms and significant cognitive impairment. For the past 10 years, this individual has expressed a plan to pursue medical-assisted death. To date, this individual has not been able to pass the MAID capacity requirements. Case 3: A 72-year-old with moderate motor symptoms and declining cognitive abilities. This individual expressed an interest having MAID in the next year, but is not yet ready to make the request. Conclusions: These cases highlight the clinical and emotional challenges for patients, families, and professional staff members related to assessing the MAID eligibility criteria of being in an advanced state of decline with a reasonably foreseeable death, while still possessing the cognitive ability needed to request and consent to MAID.

\section{Poster \#25}

Parents' Perspective on the Challenges of Disclosing Huntington's Disease Information to Their At-Risk Children

C. Clare A. Gibbons ${ }^{1,2}$, Wendy Medved ${ }^{3}$, Wendy S. Meschino $^{1,4}$, Arlette Lefebvre ${ }^{5,6}$, Wai Lun Alan Fung ${ }^{1,6,7}$, Jennifer Semotok ${ }^{2}$

${ }^{1}$ Genetics Program, North York General Hospital, North York, Ontario, Canada

${ }^{2}$ Department of Molecular Genetics, University of Toronto, Toronto, Ontario, Canada

${ }^{3}$ Canadian Institute for Health Information, Toronto, Ontario, Canada

${ }^{4}$ Department of Paediatrics, University of Toronto, Toronto, Ontario, Canada

${ }^{5}$ Hospital for Sick Children, Toronto, Ontario, Canada

${ }^{6}$ Department of Psychiatry, University of Toronto, Toronto, Ontario, Canada

${ }^{7}$ Department of Psychiatry, North York General Hospital, North York, Ontario, Canada

\section{Abstract}

Background: Parents with children at risk for Huntington's disease (HD) must decide when and how to disclose HD to their children.

Objectives: To explore parents' perceived challenges and plans for disclosure to their children.

Methods: Semi-structured interviews were conducted with 11 parents of children (aged 6-21 years) at risk for HD. The constant comparative method was used for qualitative data analysis.

Results: Parents indicated that their readiness to disclose was influenced by child's level of comprehension, exposure to someone with HD symptoms, child asking questions related to $\mathrm{HD}$, need to reduce negative impact/ protect the child, and child's personality. Most parents felt that gradual disclosure was best, especially if the parent had visible symptoms. Parents were concerned that the child might worry about their parent's health, causing a negative impact on the parent-child relationship. They were also concerned about the child's reaction to learning about their own risk to develop HD and the chance that they might perceive themselves as sick or dying. Most parents' plans for disclosure included the message of hope and promise of research and progress. In some cases, the need to provide reassurance to the child caused some distortion of the implications of inheriting HD.

Conclusions: Parents are seeking advice on the best approach to disclose HD information, but they should be encouraged to assess their specific situation guided by the themes identified through these interviews.

\section{Poster \#26}

Development of a Novel Patient-Reported Outcome Measure for Huntington's Disease: The Huntington Disease Health Index (HD-HI) Study

Alistair Glidden ${ }^{1}$, Elizabeth Luebbe ${ }^{2}$, Molly Elson ${ }^{1}$, Steven Goldenthal $^{1}$, E. Ray Dorsey ${ }^{1,2}$, Chad Heatwole ${ }^{2}$

${ }^{1}$ Center for Health and Technology, University of Rochester, Rochester, NY, USA

${ }^{2}$ Department of Neurology, University of Rochester, Rochester, NY, USA

\begin{abstract}
Background: Properly validated patient-reported outcome measures for use in HD clinical trials are lacking. Health indices that reflect patient outcomes have been implemented in clinical trials for other inherited disorders, including myotonic dystrophy, facioscapulohumeral muscular dystrophy, Charcot-Marie-Tooth disorder, and spinal muscular atrophy.
\end{abstract}


Objectives: To develop a patient-reported outcome measure that reflects the most important HD symptoms as assessed by affected individuals.

Methods: From 40 interviews $(n=20$ with HD; $n=20$ caregivers), we analyzed 2082 quotes regarding the symptomatic burden of HD. We subsequently developed a survey with 216 potential symptoms representing 15 symptomatic themes. We electronically distributed the survey to individuals with HD, prodromal HD, and caregivers. Participant responses determined the prevalence and severity $(0-4)$ of each symptom, which were then multiplied to generate a population impact factor. Based on these scores and other factors (e.g., responsiveness, language), we developed the initial version of the Huntington Disease Health Index (HD-HI).

Results: Of the 669 individual who consented, 389 completed the full survey $(n=96$ with HD, 60 with prodromal HD, and 233 caregivers). Respondents hailed from 9 countries and 46 states, and were, on average, 46 years old, $77 \%$ women, and $36 \%$ college-educated. Those with manifest HD had an average self-reported Total Functional Capacity score of 9.6. The symptoms with the highest population impact factor among those with HD were fear of progression (2.21), being tired (2.20), and problems concentrating (2.18). The symptomatic themes with the highest population impact factor were fatigue (1.85), emotional issues (1.82), and difficulty thinking (1.75). Responses differed among individuals with HD, prodromal $\mathrm{HD}$, and caregivers.

Conclusions: We have developed a new patient-reported health index that can be incorporated into HD therapeutic trials in the near future, pending factor analysis, test-retest reliability, and additional beta testing.

Sponsorship: Research support provided by NJ Cure and the Huntington Study Group.

\author{
Poster \#27 Award Winner for Peter Como Neuropsychology \\ Abstract \\ Predictors of Workplace Disability in a Premanifest and \\ Manifest Huntington's Disease Cohort \\ Anita M.Y. Goh ${ }^{1,2,3}$, Emily You ${ }^{1}$, Stephanie Perin ${ }^{1}$, Fiona J. \\ Clay $^{4}$, Samantha Loi ${ }^{1,2}$, Kathryn Ellis ${ }^{1}$, Terence Chong ${ }^{1}$, \\ David Ames ${ }^{1,5}$, Nicola Lautenschlager ${ }^{4}$ \\ ${ }^{1}$ Academic Unit for Psychiatry of Old Age, Department of \\ Psychiatry, The University of Melbourne, Australia \\ ${ }^{2}$ Neuropsychiatry Unit, Melbourne Neuropsychiatry Centre, \\ Royal Melbourne Hospital, Australia \\ ${ }^{3}$ NorthWestern Mental Health, Melbourne Health, \\ Melbourne, Australia \\ ${ }^{4}$ Department of Psychiatry, University of Melbourne, \\ Melbourne, Australia \\ ${ }^{5}$ National Aging Research Institute, Parkville, Australia
}

\section{Abstract}

Background: Huntington's disease (HD) is an inherited neurodegenerative disease involving motor, cognitive, and psychiatric/behavioral impairments that will eventually affect work-role functioning. Few objective data exist regarding predictors of workplace disability in HD.

Methods: We explored the predictors of work impairment and disability in a cross-sectional cohort of 656 employed people with premanifest HD (preHD) s and 316 employed people with motor-manifest HD from the Enroll-HD study.

Results: In the preHD cohort, the number of participants who reported that they had missed work because of HD was low (2.4\%). However, $12 \%$ of the study sample reported experiencing impairment whilst working as a result of preHD; $12.2 \%$ reported work-related activity impairment as a result of preHD; and $12.7 \%$ reported impairment in their overall work ability. In the manifest HD cohort, these numbers increased: $60.1 \%$ of the study sample reported experiencing impairment whilst working as a result of preHD; $79.1 \%$ reported work-related activity impairment due to HD; $60.8 \%$ reported impairment in their overall work ability; and $64 \%$ missed work time. We will report on the predictors of workplace disability from our regression analyses. Importantly, several modifiable factors were found to predict workplace disability. Specifically, good mental and physical health served as protective factors, and good physical and mental health were associated with fewer odds of experiencing workplace disability and/or to miss work time.

Conclusions: The results provide important new knowledge for the development of future targeted intervention trials to support individuals with preHD and symptomatic HD to maintain their work roles as long as possible.

\section{Poster \#28}

Randomization Authorization Flow (RAF): It's Not Just About Meeting Eligibility Criteria

Jody Goldstein, Susan Bennett, Elise Kayson

University of Rochester, Rochester, NY, USA

\begin{abstract}
Background: Randomization Authorization Flow (RAF) is a review and approval process of predetermined key data points by a clinical research team (CRT) prior to subject randomization in a clinical trial. Screening (SC) data are reviewed from different angles. The CRT consists of a project manager (PM), data manager (DM), and RAF reviewer (RR). The RAF process helps ensure enrollment of the appropriate study-specific patient population. This gestalt
\end{abstract}


review takes into account critical elements not necessarily covered by the eligibility criteria. Looking for subtle (subjective) differences between patients upfront ensures meeting primary outcomes of the study. RAF is part of the risk-management process to identify events that may affect the achievement of protocol objectives.

Objective: Our innovative RAF provides the CRT a framework to 1) randomize the appropriate study population and meet the primary outcomes; 2) minimize the screen failure rate, decreasing costs to sponsors; 3 ) control for quality screening data; and 4) identify potential risks.

Methods: At study start-up, the team predetermines specific SC data to be reviewed, areas of risk, and clinical focus. Following SC visit, the site investigator enters data in the database, external vendor data becomes available, and the site investigator reviews SC data and provides electronic sign-off. The PM and DM collaborate to review subject SC data, contact sites if required, and confirm availability of all data necessary for RR to determine eligibility. The DM notifies RR that subject is ready for review. RR reviews all key data points to ensure subject is suitable for the study, resulting in approval or denial for participation.

Results/conclusions: RAF has evolved into a robust data tool. Through RAF, the CRT can identify safety risks, resulting in subject exclusion and/or protocol modifications. RAF reviews have resulted in cleaner data, shorter close-out timelines, and improved quality of patients enrolled.

\section{Poster \#29 Invited for Platform Presentation}

Selectivity and Biodistribution of WVE-120102, a Stereopure Allele-Specific Antisense Oligonucleotide in Development for the Treatment of Huntington's Disease

Serena Hung, Meena Me, John W. Davis II, Keith Bowman, David C.D. Butler, Foram Desai, Lanikai Guo, Naoki Iwamoto, Hyun Jang, Maria Frank-Kamenetsky, Susovan Mohapatra, Mamoru Shimizu, Stephany Standley, Nenad Svrzikapa, Hailin Yang, Jason Zhang, Zhong Zhong, Chris Francis, Michael Panzara, Chandra Vargeese

Wave Life Sciences, Cambridge, MA, USA

\footnotetext{
Abstract

Background: Huntington's disease (HD) is caused by an expansion of the cytosine-adenine-guanine (CAG) triplet repeat in HTT, which results in production of mutant huntingtin (mHTT) protein; accumulation of mHTT leads to progressive loss of neurons in the brain. While studies suggest that silencing of mHTT ameliorates or reverses the symptoms of HD, silencing of wild-type HTT (wtHTT) may have detrimental long-term consequences. Therefore, therapies that can selectively lower mHTT gene transcript expression, while leaving wtHTT largely intact, may be desirable. WVE-120102 is a
}

stereopure antisense oligonucleotide (ASO) that was developed using proprietary technologies, which allow us to precisely define the stereochemistry at each phosphorothioate linkage. WVE-120102 specifically targets the mHTT mRNA transcript with the $U$ variant of single nucleotide polymorphism (SNP) rs362331. By targeting the variant associated with the pathogenic CAG expansion (>36 CAG repeats), WVE-120102 should provide selective reduction of the level of mHTT protein.

Results: In vitro preclinical studies showed that WVE120102 (compared with stereorandom analogues) induced selective RNase H1 cleavage of mHTT in cell-free assays, and selectively decreased mHTT mRNA and protein levels compared with wtHTT in patient-derived fibroblasts. In vivo preclinical time-course studies were conducted to determine the tolerability and pharmacokinetics of WVE-120102 in cynomolgus monkeys after intrathecal bolus injection. In situ hybridization ViewRNA-stained tissues showed clear nuclear and perinuclear distribution of WVE-120102 in neurons within cortical and deep gray structures up to 28 days following a single dose. These preclinical studies supported further clinical development of WVE-120102. First-in-human studies of WVE-120102 and WVE-120101 (a stereopure ASO that targets SNP rs362307) were recently initiated; these are the PRECISION-HD2 and PRECISION-HD1 trials, respectively. The designs of these Phase Ib/IIa clinical trials, which will provide proof-of-concept of the safety, tolerability, pharmacokinetics, and pharmacodynamic effects of targeted stereopure ASO therapy in patients with HD, will be discussed.

\section{Poster \#30 \\ Telemedicine Services for Huntington's Disease: Feasibility and Initial Outcomes \\ Madaline B. Harrison ${ }^{1}$, Dana L. Morrissey ${ }^{2}$ \\ ${ }^{1}$ Department of Neurology, University of Virginia Health \\ System, Charlottesville, VA, USA \\ ${ }^{2}$ For the University of Virginia Huntington's Disease Team}

\section{Abstract}

Background: Huntington's disease (HD) causes impairment in cognitive, motor, and psychological function. Managing HD requires specialized interdisciplinary care not generally available outside of major medical centers, which can be located far from patients' home communities. Travel to a specialty center can be challenging, particularly as symptoms progress, and may present a financial burden. The University of Virginia's HD clinic began providing telemedicine services in 1999 to increase access to specialty care.

Results: We have seen 37 individual patients for 75 diagnostic and follow-up visits in a variety of settings- 
local county or health department clinics $(n=7)$, community hospitals $(n=6)$, correctional facilities $(n=3)$, psychiatric hospitals $(n=1)$, long-term care facilities $(n=6)$, and homes $(n=2)$. Services provided include diagnostic evaluation, management following a new diagnosis, and continuity of care, as well as staff training and family counseling. Initial outcomes indicate that the use of telemedicine services has saved 26,714 miles and $441 \mathrm{~h}$ of travel time for our patients and families. The service prevented 26 patients from being lost to follow-up and allowed us to follow 5 patients across multiple sites to provide continuity of specialty care. Through telemedicine, our interdisciplinary team was able to coordinate care with other providers for 8 of our patients in long-term care facilities or receiving care from local primary care physicians. Lastly, telemedicine allowed us to offer first-time HD specialty care to 7 patients. Our experience demonstrates that telemedicine is feasible and effective in providing subspecialty neurological and interdisciplinary care. It has been well received by patients, families, and off-site providers. Future goals include outreach to increase care coordination and research on specific health outcomes reflecting quality of care, such as improved care coordination, quality of life, and reduction in adverse events.

Conclusions: Interdisciplinary specialty care for HD is often available only at major medical centers that cover large geographic areas, limiting access. Telemedicine brings specialty care and consultation closer to patients with HD, families, and local care providers.

\section{Poster \#31}

Barriers and Access to Long-Term Care: A Preliminary Investigation Into Best Practices of Huntington Disease Specialty Units

Hope Heller ${ }^{1}$, Stacey Barton ${ }^{2}$

${ }^{1}$ Medstar Georgetown University Hospital, Washington, DC, USA

${ }^{2}$ Washington University School of Medicine, St. Louis, MO, USA

\begin{abstract}
Background: Long-term care placement is often one of the greatest challenges families affected by Huntington's disease (HD) face. Whether it is preconceived notions about HD, lack of knowledge about HD, or some other factor, long-term care facilities are often quick to deny admission to someone with HD.
\end{abstract}

Methods: Throughout the country there are a handful of longterm care facilities with HD specialty units. This project looked into the natural questions: What are HD specialty units doing differently? What are we missing? Can these models be replicated in other communities? Visits were made by an HD social worker to a number of these specialty unit facilities to investigate what makes them so successful and to learn why are they able to take care of multiple people with HD when other facilities have stayed away from accepting even one person with HD. Levels of care, staffing, reimbursements, and other areas of day-to-day care and logistics were looked at for this project.

Results: While facilities varied in their models of HD care, two important points were present at the HD specialty care facilities: financial incentives to HD care were key, as the majority of the facilities had some source of additional funding beyond the standard Medicaid rate; and a culture of compassion, caring, and commitment to HD care among the staff and administration was present. Facilities that have provided many years of HD specialty care had lower staff turnover and direct care providers, such as certified nurse aides, and took the time to learn and care for each individual person to determine what was the best for their care.

Conclusions: Further projects in the area of long-term care access could lead to developing increased capacity of longterm care facilities in providing care to this vulnerable population and could afford patients the equal access to care that they are currently often denied.

\section{Poster \#32}

Gender Affects Factors Associated with Placement into Long-Term Care of Patients with Huntington's Disease Machteld E. Hillen ${ }^{1}$, Cheryl A. Kennedy ${ }^{1}$, Michael McCormack $^{2}$, Ketan Hirapara ${ }^{1}$, Eli Kneisser ${ }^{1}$, Barbara Fadem $^{1}$, Chiadikaobi Okeorji ${ }^{1}$

${ }^{1}$ Rutgers New Jersey Medical School, Newark, NJ, USA

${ }^{2}$ Rowan University School of Osteopathic Medicine, Glassboro, NJ, USA

\section{Abstract \\ Background: Psychiatric problems seen in patients with Huntington's disease (HD) include depression, anxiety, psychosis, disinhibition, substance misuse, and violence. These symptoms often pre-date the diagnosis of HD. Men with HD are more likely to show criminal activity than first-degree male relatives and controls. Because of their behavioral/psychiatric, as well as physical, symp- toms most patients with HD ultimately require profes- sional care in long-term care (LTC) facilities.}

Objective: To examine the relationship between sex and factors associated with placement of patients with HD into longterm care (LTC) facilities.

Methods: Records of 33 patients (15 male) with HD, who were known to our center prior to placement in a LTC facility, were reviewed. Data collected included 
sex, ethnicity, family history, age at first symptom onset, age at diagnosis, psychiatric symptoms, medication use, time from first symptom to diagnosis (TSD), and time from diagnosis to placement in LTC (TDP). Data were analyzed with $\chi^{2}$ tests and $t$-tests.

Results: We found no significant difference in sex on TSD, but TDP was significantly higher in men within 1 year of diagnosis $(p=0.0034,17 \%$ of women, $66 \%$ of men) and within 3 years of diagnosis $(p=0.047$, $39 \%$ of women, $73 \%$ of men). A 2 -tailed $t$-test showed a significant difference for mean TDP $[p=0.035$, mean $($ females $)=6.2$ years, mean $($ males $)=2.9$ years]. We found a significant difference in use of anxiolytics in men before and after entering LTC ( $p$ $=0.006$ ).

Conclusions: When compared with female patients with HD, male patients with HD are 1) placed in LTC sooner after diagnosis; 2) less likely to be taking psychoactive medication prior to placement into LTC; and 3) equally likely to be taking psychoactive medication after placement into LTC. These results suggest that timely use of psychoactive medication may have a role in delaying the need for LTC, particularly in male patients with HD.

\section{Poster \#33}

\section{Effects of Renal Transplantation on Patient with Huntington's Disease}

Lauren Holder, Erica Sweeney, Francis Walker, Christine O’Neill

Wake Forest Baptist Medical Center, Winston-Salem, NC, USA

\begin{abstract}
Background: Chronic renal failure (CRF) is a relatively uncommon disorder currently affecting about $0.2 \%$ of the population in the USA. Given the 7/100,000 prevalence of HD, approximately 60 individuals in the USA likely have both Huntington's disease (HD) and CRF. Little is known about optimal treatment.
\end{abstract}

Case report: A 56-year-old man with a 6-year history of diagnosed HD and a 4-year history of CRF eventually treated with dialysis found the process fatiguing and limiting. After reviewing his case with nephrology, it became apparent that there is no literature addressing the effects of either dialysis or renal transplantation in HD. After weighing unknowns, this man was eventually placed on the transplant list and a cadaveric match identified. Following transplantation, he experienced a significant decline in cognitive and motor function, without obvious cause. He was hospitalized twice for new psychiatric symptoms, once for an involuntary commitment, and another time for delirium. He ended up needing 24-h care at home with full assistance with activities of daily living (ADLs) for almost 1 year. After 18 months, he began to improve. Although able to perform ADLs, ambulate independently, and function at home, his new baseline was below what would have been anticipated without intervention, with significantly greater chorea.

Discussion: Renal transplantation has the potential to chronically aggravate the underlying cognitive, behavioral, and motor symptoms of HD. In this case, it is unclear if it was the surgery, surgically related stressors, antirejection therapy, or other nontransplant-related issues that were most contributory to his problematic postoperative course. His subsequent improvement indicates that some, but not all, of his post-transplant decline was reversible. Further, published reports of the interactions of renal failure, dialysis, and transplantation in HD are likely to be informative. Stress on the brain, longer recovery time, and other factors should be considered before a patient with HD undergoes any major surgery, such as a kidney transplant.

\section{Poster \#35}

Assessing Executive Function in Huntington's Disease: A Comparison Between a Virtual Reality Task and Conventional Neuropsychological Tests

Filipa Júlio $^{1,2}$, Alexandre Malhão ${ }^{2}$, Fábio Pedrosa ${ }^{2}$, Hélio Gonçalves $^{2}$, Marco Simões ${ }^{2}$, Miguel Patrício ${ }^{2}$, Mário R. Simões ${ }^{1,3}$, Marieke van Asselen ${ }^{2}$, Miguel CasteloBranco $^{2,4,5}$, Cristina Januário ${ }^{2,5,6}$

${ }^{1}$ Faculty of Psychology and Education Sciences, University of Coimbra, Coimbra, Portugal

${ }^{2}$ Institute for Biomedical Imaging and Life Sciences (IBILI), Faculty of Medicine, University of Coimbra, Coimbra, Portugal

${ }^{3}$ PsyAssessmentLab/Research Centre of the Cognitive and Behavioural Studies and Intervention Nucleus (CINEICC), Faculty of Psychology and Education Sciences, University of Coimbra, Coimbra, Portugal

${ }^{4}$ Institute of Nuclear Sciences Applied to Health (ICNAS), University of Coimbra, Coimbra, Portugal

${ }^{5}$ Faculty of Medicin, University of Coimbra, Coimbra, Portugal

${ }^{6}$ Coimbra University Hospital, Coimbra, Portugal

\section{Abstract}

Background: Impairments in executive functions are common in Huntington's disease (HD), even in prodromal and early disease stages, and are thought to significantly influence 
the patient's functional status. Reliable ecological tools to assess and predict the impact of executive dysfunction in daily performance are needed.

Objectives: To get a comprehensive picture of the everyday executive deficits reported in HD by using 2 different approaches: "EcoKitchen" (a novel nonimmersive virtual reality task) and a conventional neuropsychological test battery.

Methods: Participants were assigned to 1 of 3 groups (controls, premanifest HD, and early manifest HD) and performed a virtual-reality task with an increasing executive load that simulates daily-life-like routines usually done in a kitchen setting (EcoKitchen). Additionally, we used an extensive neuropsychological test battery predominantly encompassing executive tests (e.g., Stroop, Wisconsin Card Sorting Test) to study the overall cognitive functioning of the three groups.

Results: The results indicate that both patients with premanifest and manifest HD showed statistically significant differences in the timing and error variables considered in "EcoKitchen" when compared with controls. Interestingly, controls and premanifest participants revealed a similar performance in the conventional neuropsychological tests applied, whereas manifest HD participants showed deficits in almost all the cognitive measures used. Conclusions: The more ecological approach created to evaluate the executive functioning of $\mathrm{HD}$ patients seems to be sensitive to early deficits in this domain. Importantly, "EcoKitchen" tasks can potentially identify subtle changes in the executive functioning of premanifest individuals and differentiate them from controls - even with small groups that show a similar neuropsychological performance in an extensive set of conventional tests. This comprehensive assessment of everyday executive function in HD will hopefully contribute to a better understanding of the phenotype of this disease, and also to a better identification and management of the patients' real-life deficits.

\section{Poster \#36}

Physical Therapy and Exercise Interventions in Huntington's Disease: A Mixed-Methods Systematic Review

Deb Kegelmeyer ${ }^{1}$, Nora Fritz ${ }^{2}$, Anne Kloos ${ }^{1}$, Ashwini Rao ${ }^{3}$, Monica Busse ${ }^{4}$, Lori Quinn ${ }^{5}$

${ }^{1}$ The Ohio State University, Columbus, OH, USA

${ }^{2}$ Wayne State University, Detroit, MI, USA

${ }^{3}$ Columbia University Medical Center, New York City, NY, USA

${ }^{4}$ Cardiff University, Cardiff, UK

${ }^{5}$ Teacher's College, Columbia University, New York City, NY, USA

\section{Abstract}

Background: Numerous studies over the past 15 years have evaluated the effects of exercise and physical therapy rehabilitation in individuals with Huntington's disease (HD). However, a comprehensive systematic review of these studies is lacking.

Methods: We conducted a mixed-methods systematic review of exercise and physical therapy interventions on physical and behavioral outcomes, as well as client-caregiver centered experiences, using the Joanna Briggs Institute guidelines. Twenty-one studies (18 quantitative and 3 qualitative, including 622 participants) were reviewed.

Results: Exercise interventions were feasible and safe to administer in people with HD. The majority of studies focused on interventions to improve mobility, balance, and fall risk, often utilizing combined aerobic and strength exercise. The most common outcome measures were gait velocity ( 8 studies), balance and balance confidence (7 studies), and the United Huntington's Disease Rating Scale Total Motor Score (TMS; 5 studies). Improvements were seen in balance (6/7 studies), gait speed ( $4 / 8$ studies), TMS (3/5 studies), pulmonary function ( $2 / 2$ studies), and quality of life ( $3 / 7$ studies). Most studies had relatively low samples sizes and were conducted over a short duration (3 months or less) with a lack of long-term follow up. Qualitative analysis revealed that people with HD and their caregivers perceived exercise as beneficial in both physical and social domains.

Conclusions: This systematic review provides evidence from 21 studies with low-to-moderate risk of bias that exercise and physical therapy may improve balance, gait speed, and motor function in people with HD. There is a need for high-quality, randomized controlled trials of exercise interventions in people at all stages of HD, as well as evaluation of mode, frequency, and intensity of exercise to effectuate disease modification or attenuate disease progression.

\section{Poster \#37}

The Role of CAG Repeat Size and Parental Sex in Huntington's Disease

Eric Keller ${ }^{1}$, Michael McCormack ${ }^{2}$, Machteld Hillen ${ }^{3}$

${ }^{1}$ Rutgers University, New Brunswick, NJ, USA

${ }^{2}$ Rowan University, Glassboro, NJ, USA

${ }^{3}$ Rutgers-NJMS, Newark, NJ, USA

\begin{abstract}
Background: Huntington's disease (HD) alleles with full penetrance are defined as alleles with $>39$ repeats. Pathological evaluation in patients with 40 and 41 repeats have shown changes consistent with HD, even when patients were clinically asymptomatic. Alleles transmitted from the male parent expand significantly when compared with alleles transmitted from the female parent. Studies of juvenile-onset HD demonstrated that $80 \%$ of juvenile cases were inherited from the male parent.
\end{abstract}


Objectives: To identify the relationship of CAG mutation repeat size with age of onset and rate of progression of HD symptoms and to identify the influence of transmitting parental sex on the CAG mutation repeat size.

Methods: Records of a sample of 73 nonrelated subjects with genetically confirmed HD were reviewed at the Samuel L. Baily HD Center at Rutgers-RWJMS ( $n=$ $49)$ and Rutgers-NJMS $(n=24)$. Data collected for this study included gene test results, age of disease onset, Total Function Score, and Chorea Score of the Unified Huntington's Disease Rating Scale (UHDRS) Total Motor Score (UHDRS-Ch) at time of diagnosis and 1 year later, family history, sex of the transmitting parent, and sex of the subject. Data were analyzed and statistical significance of associations was determined by use of the Pearson product-moment correlation coefficient.

Results: Analysis of a sample of HD-affected individuals' CAG repeat numbers displayed a strong negative correlation between CAG number and age of onset $(\mathrm{r}=-0.7947, p<$ 0.0001 ), and a significant negative correlation between age of onset of patient and the sex of the HD-transmitting parent $(\mathrm{r}=-0.407, p=0.00019)$. We found significant associations between the UHDRS-Ch with the CAG repeat size and the transmitting parent.

Conclusions: Our study confirmed that CAG repeat size is correlated with age of onset. In addition, our data support sex differences in HD despite its autosomal dominant inheritance pattern and may indicate an extragenic effect (i.e., pleiotropy or epigenetic).

\section{Poster \#38}

"Friends with Wheels": Free Transportation Pilot Helps Improve Care

Amy Chesire $^{1}$, Shari Kinel ${ }^{2}$, Kristin Strazdins ${ }^{2}$

${ }^{1}$ University of Rochester, HDSA Center of Excellence, Rochester, NY, USA

${ }^{2}$ Huntington Study Group (HSG), Rochester, NY, USA

\footnotetext{
Abstract

Background: "Friends with Wheels" (FwW) is aimed at improving care for patients with Huntington's disease (HD) by providing free, round-trip, door-to-door transportation to clinic visits and support groups.

Methods: A HD clinic identified patients who did not attend regular clinic visits and indicated difficulty for existing patients to attend follow-up visits owing to lack of transportation. The HSG received a 1-year grant from Excellus New York. We contracted with transportation services and advertised FwW at the clinic/support group. We received feedback from the clinic and patients through a telephone interview.
}

Results: From 13 June 2016 to 3 August 2017, 15 people used FwW for 29 successful clinic visits and 7 non-HD clinic visits, and 5 people attended support group meetings. FWW covered 2355 miles (ranging from 2 to 324 miles from the clinic). There were 36 scheduled visits, 5 cancellations, and 1 patient died (from advanced stage HD). Prior to FwW, patients did not regularly attend clinic/support group owing to their inability to drive or take public transportation because of disease progression, weather, and/or distance to the clinic/support group, and/or lack of an available caregiver. FwW showed improvement as follows: Scenario 1: 3 patients who did not attend regular appointments attended appointments every 4 to 8 weeks and their medicine management improved. Scenario 2: 4 patients who had not been in the clinic for 1 to 4 years became re-engaged and resumed regular appointments. Scenario 3: 2 patients who were reluctant to drive attended their first and follow-up appointments in a timely manner. Scenario 4: a patient whose children were unable to drive attended appointments regularly. Scenario 5: 5 patients attended support group meetings at night.

Conclusions: FwW improved attendance at clinics/support groups. Patients reconnected with HD specialists to help improve overall care and management of their HD. Patients enjoyed privacy, quality, and peace of mind with the personalized driver service.

\section{Poster \#39}

The Impact of Family History on the Clinical Features of Huntington's Disease

Gabe Kringlen ${ }^{1}$, Lisa Kinsley ${ }^{2}$, Sharon Aufox ${ }^{3}$, Gerald Rouleau ${ }^{4}$, Danny Bega ${ }^{5}$

${ }^{I}$ Department of Medical Genetics at Sanford Health, Fargo, ND, USA

${ }^{2}$ Department of Neurology at Northwestern University Feinberg School of Medicine

Chicago, IL, USA

${ }^{3}$ NUgene Project, Northwestern University Feinberg School of Medicine, Chicago, IL, USA

${ }^{4}$ Biostatistics Collaboration Center, Northwestern University Feinberg School of Medicine

Chicago, IL, USA

${ }^{5}$ Department of Neurology at Northwestern University Feinberg School of Medicine, Chicago, IL, USA

\section{Abstract}

Background: Huntington's disease (HD) is an autosomal dominant neurodegenerative disorder. In most cases, the disease is inherited from a parent, although a considerable number of affected persons have no reported family history of the disease. While CAG repeat length is negatively correlated with age of symptom onset, variability 
exists suggesting that other variables may influence symptom onset.

Objectives: The objective of this study is to determine whether awareness of a family history of HD has an impact on symptom onset and disease manifestations.

Methods: Data were obtained from Enroll-HD to compare subjects with a family history of HD to subjects without on various key clinical outcomes. In addition, multiple regressions were performed to investigate the impact of family history on the age at onset of depression and motor symptoms.

Results: In total, 4285 gene-positive patients were included in the analysis, of whom $4.8 \%$ had a negative family history. Controlling for CAG repeat length, a positive family history predicted an onset of depression 11.438 years earlier and an onset of motor symptoms 6.681 years earlier when compared with having a negative family history. Patients with a positive family history were more likely to report behavioral manifestations as the initial major symptom of HD (38.6\% vs 29.6\%; $p=0.023)$ and were more likely to report previous suicidal ideation/ attempts $(26.2 \%$ vs $20.3 \%$; $p=0.046)$.

Conclusions: A positive family history of HD appears to be associated with an earlier onset of depression and overall disease manifestations. Implications regarding the role of genetic versus environmental contributions to symptom onset in $\mathrm{HD}$ are discussed.

\section{Poster \#40}

Topotecan, a Topoisomerase-1 Inhibitor, Retards the Disease Pathogenesis in a Mouse Model of Huntington's Disease

Shashi Shekhar Kumar, Naman Vatsa, Vipendra Kumar, Brijesh Kumar Singh, Imran Jamal, Ankit Sharma, Nihar Ranjan Jana

Cellular and Molecular Neuroscience Laboratory, National Brain Research Centre, Manesar, Gurgaon, Haryana India

\footnotetext{
Abstract

Background: Huntington's disease (HD) is a fatal and late-onset progressive neurodegenerative disorder with an autosomal dominant manner of inheritance and caused by the abnormal expansion of CAG repeats in the exon- 1 of the HD gene. One of the common pathological hallmarks of HD is the accumulation of mutant huntingtin as insoluble aggregates that can be seen predominantly in the nucleus and to a lesser extent in other subcellular structures. After the discovery of the HD gene, considerable progress has been made in understanding the disease pathogenesis, and multiple drug targets have been identified, even though currently there is no effective therapy.
}

Results/conclusions: Here, we demonstrate that the treatment of topotecan (a Food and Drug Administration-approved chemotherapeutic agent), a brain-penetrating topoisomerase-1 inhibitor to HD transgenic mice, considerably improved their motor behavioral abnormalities along with significant extension of lifespan. Improvement of behavioral deficits is accompanied by the significant rescue of their progressively decreased body weight, brain weight, and striatal volume. Interestingly, topotecan treatment also significantly reduced insoluble mutant huntingtin load in the HD mice brain. Finally, we show that topotecan treatment of HD mice not only inhibits the expression of transgenic mutant huntingtin, but also at the same time induces the expression of Ube3a, an ubiquitin E3 ligase linked with the clearance of mutant huntingtin. These finding suggests that topotecan could be a potential therapeutic molecule to delay the progression of $\mathrm{HD}$.

\section{Poster \#41 \\ The Unified Huntington's Disease Rating Scale Total Motor Score in Prodromal Huntington's Disease \\ Douglas Langbehn ${ }^{1}$, Bernard Ravina ${ }^{2}$, Steven Hersch ${ }^{2}$ \\ ${ }^{1}$ University of Iowa, Carver College of Medicine, Iowa City, IA, USA \\ ${ }^{2}$ Voyager Therapeutics Inc., Cambridge, MA, USA}

\section{Abstract}

Background: Delay or prevention of future illness is the ultimate therapeutic goal for prodromal Huntington's disease (HD). Approaches to testing a therapy could use either progressive longitudinal measures of prodromal HD or the occurrence of an imprecise diagnostic milestone. Despite considerable research, efficacy measures for prodromal HD continue to pose important challenges to developing therapies.

Methods: We compared the properties of potential outcomes in individuals with presymptomatic (preHD) using data from Enroll-HD and COHORT in longitudinal models relevant to clinical trial design. We compared these with published results from TRACK-HD, PREDICT-HD, and PHAROS.

Results: In preHD, the Unified Huntington's Disease Rating Scale Total Motor Score (TMS) consistently demonstrated the strongest signal-to-noise ratio (effect size) among all clinical measures studied, including multiple measures of cognition and of mixed cognitive and motor ability such as the Symbol Digit Modality Test (SDMT). In Enroll, among the $40 \%$ at highest risk for imminent diagnosis, the second-largest longitudinal decline was for Stroop Word. Its signal-to-noise was $43 \%$ of the TMS signal. In preHD, the TMS also outperformed recently proposed composite measures that are optimized for early diagnostic stages. Within the same high-risk preHD segment, signal-to-noise was $73 \%$ to $88 \%$ of the TMS, 
depending on the composite definition. PreHD SDMT scores did not decline in Enroll or COHORT; however, there was less practice effect improvement than in controls.

Discussion: Among potential longitudinal outcomes for prodromal HD, the TMS has superior measurement properties. Existing cognitive or composite outcomes would require notably larger sample sizes or treatment effects. Practice effects further complicate the use of cognitive measures in preHD trials and substantially cloud estimation of realistic treatment effects. The TMS alone may not be sufficient as a measure of progression, since motor signs are also susceptible to symptomatic therapies. Biomarkers or an assessment of treatment discontinuation could provide additional supportive data.

Conclusions: We are still uncertain how to effectively measure prevention of clinical HD onset in a trial that is short enough to be feasible. We have assessed evidence from multiple studies to demonstrate that increases in subtle motor signs are detected more reliably than subtle cognitive changes. We nonetheless recognize that use of motor symptoms alone may not be sufficient, and we encourage further discussion in light of the now-extensive cumulative evidence.

\section{Poster \#42}

Factors That Influence Financial Decision Making in Huntington's Disease

Sarah Mason ${ }^{1}$, Wei-Li Kuan ${ }^{1}$, Roger Barker ${ }^{1}$, Michelle Baddeley ${ }^{2}$

${ }^{1}$ John Van Geest Centre for Brain Repair, Cambridge, UK

${ }^{2}$ Institute of Choice - UniSA, North Sydney, Australia

\begin{abstract}
Background: Advances in technology have changed the way in which we spend and manage our money. For most people this has been a liberating development, but for the thousands of people living with dementia this poses serious challenges to their ability to live independently and puts them at increased risk of financial abuse. A loss of financial autonomy and monetary mismanagement is a characteristic feature of Huntington's disease (HD); however, it is currently unclear what drives these problems. We know that patients experience disruption to the functional integrity of the frontostriatal circuitry, leading to a characteristic dysexecutive syndrome that can be accompanied by behavioral problems, such as impulsivity, poor risk assessment, and emotional changes. There is also evidence that they can struggle to interact socially, but the extent to which these problems relate to their financial vulnerability is unknown.
\end{abstract}

Methods: In this study, we adopted both theory and methodology from the field of social economics to help provide insight into the reasons why patients with HD are vulnerable to financial abuse.

Results: The results indicate that patients' generosity on social economics tests, such as the Dictator, Ultimatum, Trust, and Public Goods Games, increases with advancing HD, whereas their ability to adjust their risk-taking behavior in the context of relevant information deteriorates. Furthermore, performance strongly correlates with their ability to accurately interpret the feelings and beliefs of their confederates.

Conclusions: This suggests that both an increased tendency to make risky decisions and difficulty identifying spurious financial offers or disingenuous people may be important factors that promote the susceptibility of patients with HD to financial abuse.

\section{Poster \#43 \\ Biological Motion Perception in Huntington's Disease Tamara Matheis $^{1}$, Lisa Muratori ${ }^{1,2}$, Ralf Reilmann ${ }^{1,3}$ ${ }^{1}$ George Huntington Institut, Muenster, Germany \\ ${ }^{2}$ Rehabilitation Research \& Movement Performance Laboratory, Departments of Physical Therapy and Neurobiology, Stony Brook University, NY, USA \\ ${ }^{3}$ Department of Neurodegenerative Diseases and Hertie Institute for Clinical Brain Research, University of Tuebingen, Tuebingen, Germany}

\begin{abstract}
Background: The ability of healthy individuals to detect biological motion by using a small number of moving points is well established. While impairments in slowed saccadic eye movements and visual fixation, visuospatial dysfunction, and poor contrast sensitivity are well-known issues in patients with Huntington's disease (HD), there is little work done about perception of biological motion (BM) in HD. A pilot study in 2016 detected a decline of this ability in persons with HD. Similar to volitional saccades, which show even deficits in prediagnostic HD, impairments in perception of biological motion might serve as an early marker for disease progress. Moreover, visual perception is likely to have a direct relationship to motor learning and control. So detecting problems in visual perception of patients with HD might help to understand typical behavior patterns.

Objectives: To investigate if impairments in perception of biological motion exist in patients with HD in comparison to healthy peers.

Methods: Biological motion animations have been created by filming healthy and impaired persons in movement using a Vicon Motion System. Those were displayed as 13-point full-body marker sets (point-of-light images) on a computer screen. When presented to the participants, the point-of-light
\end{abstract}


images were superimposed by 400 randomly moving points that decline over time. Participants had to distinguish between 3 different movements, and whether the biological motion was impaired or healthy. The point-of-light images were displayed in 42 trails in random order. The participants with HD were compared with healthy peers in the ability to accurately define the image motion and health type. Presented motions included walking, sit and stand, and stair climbing; health types included healthy and Parkinson's disease.

Results: Patients with HD show a significant decreased ability to accurately perceive biological motion compared with healthy controls. The number of correct detections of movements across health types was significantly higher in control subjects. The stair-climbing motion showed the largest effect; participants with HD had more difficulty guessing both the movement and health type correctly. The control group showed a significantly increased learning effect during the 42 trials, compared to the HD group.

Conclusions: Using BM appears to be a suitable method to examine the perception of persons with HD in comparison to healthy peers and may therefore serve as an early biomarker. Patients with HD have problems in perceiving biological motion as well as healthy persons. As motion perception drives action, errors in perception may lead to errors in motion, and these perception errors may serve as early biomarkers for disease onset and progression.

\section{Poster \#44}

Presence of Tau Pathology Within Fetal Neural Allografts in Patients with Huntington's and Parkinson's Disease

Giulia Cisbani ${ }^{1}$, Alexander Maxan ${ }^{1}$, Jeffrey Kordower ${ }^{2}$, Emmanuel Planel ${ }^{1,3}$,

Thomas Freeman ${ }^{4,5}$, Francesca Cicchetti ${ }^{1,3}$

${ }^{1}$ Centre de Recherche du CHU de Quebec, Axe

Neurosciences, Quebec, Canada

${ }^{2}$ Department of Neurological Sciences and Center for Brain

Repair, Rush University Medical Center, Chicago, IL, USA

${ }^{3}$ Departement de Psychiatrie \& Neurosciences, Université

Laval, Quebec, Canada

${ }^{4}$ Department of Neurosurgery and Brain Repair, University of

South Florida, Tampa, FL, USA

${ }^{5}$ Center of Excellence for Aging and Brain Repair, University

of South Florida, Tampa, FL, USA

\section{Abstract}

Background: Cell replacement has been explored as a therapeutic strategy to repair the brain in patients with Huntington's and Parkinson's disease (HD and PD, respectively). Postmortem evaluations of healthy grafted tissue in such cases have revealed the development of Huntington- or Parkinsonlike pathology, including mutant huntingtin aggregates and
Lewy bodies. An outstanding question remains if tau pathology can also be seen in patients with HD and PD who had received fetal neural allografts.

Methods: This was addressed by immunohistochemical/ immunofluorescent stainings performed on grafted tissue of 2 patients with HD, who came to autopsy 9 and 12 years posttransplantation, and 2 patients with PD who came to autopsy 18 months and 16 years post-transplantation.

Results: We show that grafts also contain tau pathology in both types of transplanted patients. In 2 patients with HD, the grafted tissue showed the presence of hyperphosphorylated tau [both AT8 (phospho-tau Ser202 and Thr205) and CP13 (pSer202) immunohistochemical stainings)] pathological inclusions, neurofibrillary tangles, and neuropil threads. In patients with PD, the grafted tissue was characterized by hyperphosphorylated tau (AT8; immunofluorescent staining) pathological inclusions, neurofibrillary tangles, and neuropil threads, but only in the patient who came to autopsy 16 years post-transplantation. Abundant tau-related pathology was observed in the cortex and striatum of all cases studied. While the striatum of the grafted patients with HD revealed an equal amount of 3repeat and 4-repeat isoforms of tau, the grafted tissue showed elevated 4-repeat isoforms by Western blot.

Conclusions: This suggests that transplants may have acquired tau pathology from the host brain, although another possibility is that this was due to acceleration of aging. This finding not only adds to the recent reports that tau pathology is a feature of these neurodegenerative diseases, but also that tau pathology can manifest in healthy neural tissue transplanted into the brains of patients with 2 distinct neurodegenerative disorders. We provide the first evidence for the presence of tau pathology in healthy tissue transplanted to patients with HD and PD more than a decade postgrafting. This is extremely important to the HD community as it extends the growing body of evidence that protein transfer is common to many neurodegenerative disorders, and suggests a possible target for future treatments or interventions.

\section{Poster \#45}

Cross-Sectional Exploratory Study of D-Serine Levels in Huntington's Disease

Andrew McGarry ${ }^{1}$, Basant Pradhan ${ }^{1}$, Irving Wainer ${ }^{2}$

${ }^{1}$ Cooper University Healthcare at Rowan University, Department of Neurology, Camden, NJ, USA

${ }^{2}$ National Institute on Aging, Bethesda, MD, USA

\section{Abstract}

Background: D-serine (DSR), the most biologically active D-amino acid in the mammalian brain, plays a significant role in glutamate signaling, dendritic development, synaptic 
plasticity, and neuronal migration. It is an obligatory coagonist of the $N$-methyl-D-aspartate (NMDA) receptor (NMDAR) by binding potently to the NMDA-associated glycine modulatory site. DSR is highly expressed in brain regions enriched with NMDAR, such as cortex, hippocampus, thalamus, hypothalamus, and amygdala. It is active at synaptic NMDAR, where the most common forms of plasticity, long-term potentiation, and depression are mediated. Strict regulation of NMDAR activity is also thought to be relevant to neurotoxicity. NMDA hyperactivity has been implicated in cell death for several neurodegenerative disorders; conversely, NMDAR hypoactivity promotes apoptosis and contributes to mild cognitive impairment and psychosis/ cognitive impairment in schizophrenia. Little is known about DSR biology in HD. It is not clear if DSR helps potentiate HD toxicity, can serve as a biomarker of onset or progression, or facilitates an adaptive response at synaptic NMDAR receptors that may be amenable to therapeutic amplification.

Methods: In this pilot study, we quantify DSR levels in plasma and cerebrospinal fluid in HD, and correlate data to clinical severity as measured by the Unified Huntington's Disease Rating Scale. We will sample 15 subjects at a single time point from different disease stages (HD1, HD2, HD3).

Results: The first study visits were on 1 September 2017. Data will be analyzed once all subjects have participated.

Conclusions: In a context where no specific treatment exists for cognitive decline in HD, it is intriguing to consider that DSR may influence synaptic plasticity toward improved cognitive performance. Data will serve to guide development of several projects, including a larger prospective study aimed at tracking DSR over longer periods and pilot projects aimed at testing DSR, alone or in combination with other molecules, as a therapeutic agent in HD.

\section{Poster \#46}

Huntington's Disease Regulatory Science Consortium: Enabling Pathways to Effective Treatments Through Regulatory Science and Innovation

Cheryl Fitzer-Attas ${ }^{1}$, Diane Stephenson ${ }^{2}$, Klaus Romero ${ }^{2}$, Volker D. Kern' ${ }^{2}$ Cristina Sampaio ${ }^{1}$

${ }^{1}$ CHDI Foundation, Princeton, NJ, USA

${ }^{2}$ Critical Path Institute, Tucson, AZ, USA

\section{Abstract}

Background: There are few approved medications for the treatment of Huntington's disease (HD), so there is a significant and urgent need for effective new therapies. Recent progress in understanding disease pathophysiology, identification of new therapeutic targets, and insights derived from technological developments are driving new pharmacotherapeutic approaches in HD. This is evident in the significant increase in pharmaceutical companies now advancing therapies for HD. Therefore, Critical Path Institute (C-Path) and the Cure Huntington's Disease Initiative (CHDI) Foundation have formed a collaboration to bring together the HD community and build a foundation for the Huntington's Disease Regulatory Science Consortium (HD-RSC).

Objectives: To establish a broad-based consortium, the HDRSC, which will advance disease-specific data standards and drug development tools, as well as define regulatory pathways to speed the approval of HD therapeutics.

Methods: HD-RSC will bring together partners from industry, academia, government, and patient advocacy organizations to share data and expertise to achieve regulatory goals in support of HD drug development. Ongoing work, including creating an inventory of clinical data from observational cohorts and clinical trials and existing computational models, will serve as a foundation for HD-RSC regulatory science initiatives. C-Path's core competencies are being leveraged for the development of Clinical Data Interchange Standards Consortium (CDISC) data standards for HD, and development of a secure, online database to house standardized clinical data from new and existing trials and observational cohorts.

Results: Data sharing among consortium members will enable the development of quantitative simulation platforms that integrate disease, drug, and clinical trial features as tools to select optimal trial designs, as well as efforts to qualify biomarkers and clinical outcome assessments for HD trials.

Conclusions: The short-term goal of HD-RSC will be to bring together the HD community; report progress on the foundational goals of the C-Path and CHDI collaboration; and to plan the way forward for a global, unified, data-driven initiative to enable efficient advancement of new therapies.

\section{Poster \#47 \\ The Food and Drug Administration is Listening: Integrating the Voice of the Patient in Drug Development for Parkinson's and Huntington's Diseases \\ Diane Stephenson ${ }^{1}$, Klaus Romero ${ }^{1}$, Gerald Podskalny ${ }^{2}$, Eric Bastings $^{2}$, Billy Dunn ${ }^{2}$, Susanne Goldstein ${ }^{2}$, Theresa Mullin ${ }^{2}$ ${ }^{1}$ Critical Path Institute, Tucson, AZ, USA \\ ${ }^{2}$ U.S. Food and Drug Administration (FDA), Silver Spring, $M D$, USA}

\section{Abstract}

Background: Traditional drug development has not systematically incorporated patients' perspectives and preferences into the process. In order to address this need, the 
Food and Drug Administration's (FDA) Patient-Focused Drug Development (PFDD) meetings have been organized to more systematically gather patients' perspectives on their condition and available therapies. On 22 September 2015, the FDA hosted a public PFDD meeting focused on HD and PD to gain patient feedback.

Objectives: To present highlights from the PFDD meetings held at FDA for Parkinson's disease (PD) and Huntington's disease (HD) to raise awareness of the importance of listening to the needs of those living with movement disorders.

Methods: One hundred participants attended the FDA's PFDD meeting for PD in person and $>160$ joined the 4-h PD segment of the meeting remotely. Two-thirds of the participants who attended in person had a diagnosis of PD. Fifty participants attended the HD PFDD meeting in person and 60 participants provided input through the live webcast. The FDA heard directly from participants with an emphasis on symptoms, existing treatment options, and the unmet need for new therapies.

Results: Participant comments focused on the disease symptoms that impact their daily activities most. In addition, participants presented comments from the patients' perspective on treatments of their disease. Gathering of public input was extended for 2 months past the meeting; all feedback from the meeting and extension period was compiled to prepare a voice-of-the-patient report for HD and PD that are publicly available. This information will be integrated into evaluation of improved outcome measures and assessment of risk/benefit in future trials.

Conclusions: Engaging patients in a public forum fosters a thorough understanding of the impact caused by the diverse symptoms of these diseases from the patient perspective. The FDA's PFDD initiative promises to identify unmet needs and aid in the FDA's decision-making as it establishes the context in which it considers the risks and benefits associated with future treatments for HD and PD. This aligns with the goals of Critical Path Institute's two precompetitive consortia Critical Path for Parkinson's (CPP) and Huntington's Disease Regulatroy Science Consortium (HD-RSC) aimed at accelerating development of effective treatments through robust collaboration centered on data sharing, patient input and regulatory science.

\section{Poster \#48}

Factors Influencing Completion of Predictive Testing for Huntington's Disease

Margi Patel ${ }^{1}$, Mahvish Ayman ${ }^{2}$, Victor Sung ${ }^{1}$

${ }^{1}$ University of Alabama (UAB), Birmingham, AL, USA

${ }^{2}$ Shadan Institute of Medical Sciences, Hyderabad, Telangana, India

\section{Abstract}

Objectives: To determine if the new simplified testing protocol led to an increase in completion rate of predictive testing for Huntington's disease (HD), and to study factors influencing testing process. HD, an autosomal dominant disorder, presents with disabling cognitive and motor symptoms. At-risk individuals can be tested (according to existing national guidelines) before clinical symptoms develop, but testing can be stressful, resulting in up to $40 \%$ incompletion rates.

Methods: In January 2014, testing protocol at the University of Alabama's HD clinic was changed, requiring 3 in-person visits (genetic counseling via telephone and psychiatric evaluation via mailed questionnaire) as compared with 5 visits previously. The study population $(n=$ 46) comprised patients who began predictive testing at our HD clinic between August 2009 and October 2015. A chart review was done to collect variables affecting completion of testing. A survey regarding variables was emailed to patients whose email addresses were found $(n=11)$.

Results: A little fewer than half (54.4\%) of patients completed the testing before the protocol change and $48.8 \%$ completed it after the protocol change. Individual variables, like sex, age, distance from clinic, number of living relatives with $\mathrm{HD}$, motivation for testing, education, and marital status, were compared, and no statistical significance was achieved for any of the above.

Conclusions: The primary hypothesis that the completion rate would be increased after the protocol change was rejected. The primary motivation for testing and proximity to clinic (secondary hypotheses) were not statistically significant. Limitations to our study include small scale and loss to follow-up. More studies are needed to determine if national guidelines for HD testing need to change for more accessibility to patients.

\section{Poster \#49 \\ Dysregulated Amino Acid Homeostasis and Signaling in Huntington's Disease: Therapeutic Opportunities \\ Bindu D. Paul, Juan I. Sbodio, Solomon H. Snyder \\ The Solomon H. Snyder Department of Neuroscience, Johns Hopkins University School of Medicine, Baltimore, MD, USA}

Abstract

Background: Perturbations in amino-acid metabolism have been reported in Huntington's disease (HD), but their origins have remained unclear. We show here that aberrant regulation of activating transcription factor 4 (ATF4), the master regulator of amino-acid biosynthetic and transport genes, is responsible for the deficit. ATF4 
is induced in wild-type striatal cells, but not in HD cells, when cells are subjected to cysteine deprivation. The abnormality stems from the elevated oxidative stress in HD caused by defective thiol metabolism, which creates a toxic feed-forward cycle to prevent optimal stress responses of striatal cells. Mitigating oxidative stress using ascorbate restores ATF4 function and rescues response to stress stimuli such as nutrient deprivation.

Results: Our studies identify the ATF4 regulatory network as a node for therapeutic intervention. Accordingly, agents that stimulate the induction and activity of ATF4 would be beneficial in HD. We discuss compounds that activate the ATF4 and promote neuronal health in HD. Our findings identify a molecular link between redox imbalance and metabolic dysfunction during neurodegeneration in HD.

\section{Poster \#50}

Feasibility and Acceptability of Implementing a ClinicBased Physical Activity Coaching Intervention in People with Premanifest and Early-Stage Huntington's Disease Lori Quinn ${ }^{1}$, Karen Marder ${ }^{2}$, Monica Busse ${ }^{3}$, Ciarán Friel ${ }^{1}$, Manuela Caciula ${ }^{1}$

${ }^{1}$ Teachers College, Columbia University, NY, NY, USA

${ }^{2}$ Columbia University, NY, NY, USA

${ }^{3}$ Cardiff University, Cardiff, UK

\begin{abstract}
Background: While there is increasing recognition of the importance of exercise and physical activity in people with Huntington's disease (HD), we do not yet have an understanding of the optimal methods to facilitate exercise adherence within clinic settings. We have previously developed a physical activity coaching program, Engage-HD, for people with early-to-mid-stage HD, which was found to be feasible and acceptable, and resulted in improvements in physical activity, self-efficacy, and cognition. However, research from other neurodegenerative diseases such as Parkinson's disease suggests that exercise interventions have the potential to be most effective in either delaying disease onset or slowing disease progression when implemented at the earliest stages of the disease.

Methods: This study will evaluate the feasibility and acceptability of implementing a physical activity coaching program for people with premanifest and early-stage HD [Total Functional Capacity (TFC) 10-13] in a clinic setting. We will recruit 7 individuals with premanifest HD and 7 individuals with early-stage manifest HD (TFC 10-13) at the Columbia University Huntington's Disease Society of America Center of Excellence. The Engage-HD coaching program has been modified for this population, and includes a disease-specific physical activity workbook and detailed coaching program to
\end{abstract}

be delivered by a physical therapist. Participants in this study will undergo a one-to-one physical activity coaching session in the clinic followed by 3 follow-up video/telephone calls over a 4-month period to facilitate their engagement in physical activity and exercise. Wrist-worn physical activity monitors (FitBits) will be utilized to facilitate adherence and provide participant-specific feedback. Assessment of feasibility will include recruitment, adherence, and retention rates, and acceptability of the intervention will be evaluated via questionnaires. Pre- and post-intervention assessments will explore effect estimates of disease-specific motor function, self-efficacy, cognition, physical activity, and general mobility. Results from this study will be used to make relevant adaptations to prepare for a phase II randomized trial.

\section{Poster \#51}

Influence of Cognitive and Motor Dual Tasks on Gait in Prodromal and Manifest Huntington's Disease

Ashwini Rao, Karen Marder

Huntington's Disease Center of Excellence, Columbia University, New York, NY, USA

\begin{abstract}
Background: Huntington's disease (HD) results in cognitive and motor impairments that begin in the prodromal stage and progress in manifest HD. The effect of dual task (motor and cognitive) performance on gait has not been examined in prodromal HD.
\end{abstract}

Objectives: To examine the influence of motor and cognitive secondary tasks on gait in prodromal and early manifest stages of HD.

Methods: We recruited 62 subjects for this study (18 controls, 24 with prodromal HD, and 20 with manifest HD). Gait was tested under 4 conditions: baseline walking, cognitive dual task (walking + verbal fluency task), simple manual (walking + carrying 1 glass of water on tray), and complex manual (walking + carrying 2 glasses of water on tray). Spatiotemporal gait variables were measured with the Gaitrite mat. Outcomes of interest were gait speed, stride length, double support percentage, and variability of stride length and stance time.

Results: Across all conditions, patients with prodromal and manifest HD demonstrated slower gait speed, shorter stride length, greater time in double support, and increased variability compared with controls. While performing a simple or complex manual task during gait, all 3 groups demonstrated a similar change in speed, stride length, time in double support, and gait variability. When performing a cognitive task during gait, patients with prodromal and manifest HD demonstrated greater increase in stride-length variability compared with controls. 
Discussion: Performance of concurrent manual tasks during gait resulted in a similar magnitude of changes in gait measures in patients with prodromal and manifest HD, and healthy controls. While performing concurrent cognitive tasks during gait, patients with prodromal and manifest HD demonstrated greater increase in stride-length variability compared with control subjects. In other words, we find that performing a cognitive task during walking results in highly variable stepping, which may increase future risk for falls. Given the association of stride-length variability with falls, assessment of cognitive dual task during gait may improve prediction of future fall risk in early HD. Functional mobility often requires concurrent performance of manual and cognitive tasks while walking. We examined if performing cognitive and manual tasks during walking has an effect on quantitative measures of gait.

\section{Poster \#52}

Impaired Homeostatic Plasticity in YAC128 Huntington's Disease Mouse Cortical Neurons Rescued by Pridopidine Amy Smith-Dijak ${ }^{1}$, James Mackay ${ }^{1}$, Michal Geva ${ }^{2}$, Michael Hayden $^{2}$, Lynn A. Raymond ${ }^{1}$

${ }^{1}$ Department of Psychiatry and Djavad Mowafaghian Centre for Brain Health, University of British Columbia, Vancouver, $B C$, Canada

${ }^{2}$ Research and Development, Teva Pharmaceutical Industries, Vancouver, BC, Canada, and Netanya, Israel

\begin{abstract}
Background: Homeostatic plasticity is a key mechanism for maintaining stability in brain circuits. By globally adjusting the strength while preserving relative weights of synapses, homeostatic synaptic scaling maintains neuronal activity in a physiological range, thereby facilitating new learning and mental flexibility, which are impaired early in Huntington's disease (HD). Previous work in rodent cortical neurons shows that 48-h treatment with tetrodotoxin (TTX) to block action potentials causes upscaling of excitatory glutamatergic synapses via reduced activitydependent release of brain-derived neurotrophic factor (BDNF).
\end{abstract}

Methods: Since mutant huntingtin protein reduces BDNF expression in cortical neurons, we hypothesized that: 1) cortical neurons from YAC128 HD mice have a baseline deficit in BDNF signaling and therefore reduced capacity to respond to TTX with synaptic upscaling; 2) pridopidine, suggested in the PRIDE-HD trial to stabilize total functional capacity and also shown to upregulate BDNF signaling in rodent brain (Geva, HMG 25:3975, 2016), can rescue a homeostatic plasticity deficit in YAC128 cortical neurons. Here, we made electrophysiological recordings from YAC128 and wild-type mouse cortical neuronal cultures, measuring function of glutamatergic synapses after 48h TTX versus vehicle control treatment. Results: Wild-type cortical neurons responded to action potential silencing with increased frequency and amplitude of miniature excitatory synaptic currents (reflecting synaptic numbers and strength), whereas there was no response in YAC128 cultures. Strikingly, pretreatment for $48 \mathrm{~h}$ with 0.1 to $1 \mu \mathrm{M}$ pridopidine before TTX exposure restored synaptic upscaling in YAC128 neurons. We are investigating whether pridopidine's effect is mediated by restoring basal BDNF levels in YAC128 cortical neurons. Our results shed new light on mechanisms by which pridopidine may slow decline in functional capacity in HD and potentially ameliorate cognitive deficits associated with early-stage disease.

\section{Poster \#53}

A Qualitative Analysis of the Impact of Chorea on HealthRelated Quality of Life in Huntington's Disease: Perspectives from Patients, Family Members, and Professional Providers

Carey Sherman ${ }^{1}$, Ravi Iyer ${ }^{2}$, Victor Abler ${ }^{2}$, Noelle E. Carlozzi $^{3}$

${ }^{1}$ Institute for Social Research, University of Michigan, Ann Arbor, MI, USA

${ }^{2}$ Teva Pharmaceutical Industries, Frazer, PA, USA

${ }^{3}$ Department of Physical Medicine and Rehabilitation, University of Michigan, Ann Arbor, MI, USA

\begin{abstract}
Background: Chorea, one of the prominent motor symptoms in Huntington's disease (HD), can interfere with day-to-day functioning and have a negative impact on health-related quality of life (HRQoL).
\end{abstract}

Objectives: To characterize the impact of chorea on everyday functioning and HRQoL in individuals with $\mathrm{HD}$, and to understand the similarities and differences of patient, caregiver, and provider perceptions of chorea.

Methods: Pre-existing data from focus groups of individuals with manifest HD ( $n=8$ early-stage HD; $n=16$ late-stage HD), individuals at risk for or with premanifest HD $(n=16)$, family caregivers for individuals with $\operatorname{HD}(n=17)$, and professional providers $(n=25)$ with $>3$ years' experience treating HD were collected for this analysis. Focus group recordings were transcribed verbatim and analyzed using QSR International's NVivo 11 to identify themes or patterns based on participant group.

Results: Dominant themes consistent across all groups included anticipation of disease progression and associated adverse consequences, concern over the stigma of HD, the negative effect on independence, interpersonal relationships and mental health, and potential coping strategies. 
Individuals at risk for or with premanifest HD expressed concern over future HD onset, and voiced an interest in proactively managing their disease. Participants with early-stage HD described their need to adapt to the increasing role their disease plays in their lives, and strategies aiming to maintain their current lifestyle. In contrast, participants with late-stage HD were concerned about decreased autonomy and social engagement, and potential progression of disease. Caregivers described their need to monitor changes in the person they care for, and to ensure they remained engaged and physically mobile. Providers reiterated the impact of HD on patients' social roles and also expressed particular concern regarding patient safety.

Conclusions: HD chorea significantly impacts day-to-day functioning and HRQoL across the HD disease spectrum. As such, appropriate management of chorea can potentially improve day-to-day functioning and overall HRQoL.

\section{Poster \#54}

\section{Experience of Individuals With Huntington's Disease and Chorea}

Eileen Mack Thorley ${ }^{1}$, Ravi Iyer ${ }^{2}$, Noelle Carlozzi ${ }^{3}$, Paul Wicks $^{1}$, Chris Curran ${ }^{1}$, Sanjay Gandhi ${ }^{2}$, Victor Abler ${ }^{2}$, Karen Anderson ${ }^{4}$

${ }^{1}$ PatientsLikeMe, Cambridge, MA, USA

${ }^{2}$ Teva Pharmaceutical Industries, Frazer, PA, USA

${ }^{3}$ Department of Physical Medicine and Rehabilitation, University of Michigan, Ann Arbor, MI, USA

${ }^{4}$ Georgetown University, Washington, DC, USA

\begin{abstract}
Background: Huntington's disease (HD) is characterized by motor dysfunction, cognitive impairment, and emotional-behavioral symptoms. As the hallmark feature of HD, chorea can interfere with daily functioning, negatively impact healthrelated quality of life (HRQoL), and be associated with social stigma.
\end{abstract}

Objectives: To evaluate how chorea impacts HRQoL and overall functioning among patients with HD participating on the PatientsLikeMe website (www.patientslikeme.com).

Methods: A survey was provided to participants with HD and/or their caregivers via PatientsLikeMe (9 February 2017-22 March 2017), comprising multiple-choice and open-ended questions designed to assess how chorea impacts HRQoL and overall functioning, and the importance of treating chorea. The HD HRQoL measurement system (HDQLIFE) measured patient-reported outcomes of chorea. Scores of $>60$ indicate significant reported concerns in physical and/or social functioning due to chorea. Anxiety and Stigma scores in the Patient-Reported Outcomes Measurement Information System (PROMIS)/Neuro-QoL
Measurement Systems were analyzed in participants with HDQLIFE Chorea scores $>60(n=45)$ and $<60(n=38)$.

Results: One hundred and fifteen participants ( $n=35$ caregivers; $n=80$ individuals with HD) were included in this study. Among those experiencing chorea $(n=83,72 \%$ of respondents) as a patient or caregiver, $66 \%$ indicated it was "very important" to manage chorea; however, only $47 \%$ agreed that their current medication regimen helped manage their movements. Themes identified from open-text results indicated loss of independence, mobility issues, and unpredictability of chorea as the main reasons chorea management is important. In general, respondents reported that chorea negatively affected HRQoL (mean \pm SD HDQLIFE Chorea score $59.3 \pm 6.1)$. Consistent with this, respondents with Chorea scores $>60$ had significantly higher PROMIS Anxiety ( $p=$ $0.0423)$ and Neuro-QoL Stigma $(p<0.0001)$ scores than those with scores $<60$.

Conclusions: Results highlighted the negative impact of chorea on HRQoL and overall functioning in HD individuals. Better chorea treatment options are needed to help improve HRQoL in these individuals, and patients' experience of anxiety and stigma should be considered in treatment plans.

\section{Poster \#55 \\ Physician Perceptions of Unmet Medical Needs in Huntington's Disease in the USA \\ Victor Sung ${ }^{1}$, Ravi Iyer ${ }^{2}$, Sanjay Gandhi ${ }^{2}$, Bijal Shah Manek ${ }^{3}$, Marco DiBonaventura ${ }^{3}$, Victor Abler ${ }^{2}$, Daniel Claassen ${ }^{4}$ \\ ${ }^{1}$ University of Alabama School of Medicine, Birmingham, $A L$, USA \\ ${ }^{2}$ Teva Pharmaceutical Industries, Frazer, PA, USA \\ ${ }^{3}$ Ipsos Healthcare, San Francisco, CA, USA \\ ${ }^{4}$ Vanderbilt University Medical Center, Nashville, TN, USA}

\section{Abstract}

Background: Tetrabenazine has demonstrated efficacy in treating chorea in patients with HD, but is associated with neuropsychiatric side effects, such as depression and anxiety, that may limit its clinical utility.

Objectives: To examine the unmet needs associated with tetrabenazine as a treatment for HD-related chorea.

Methods: Board-certified/board-eligible neurologists, in practice for $>5$ years and who treated $>3$ patients with HD in the past 2 years were recruited from an online physician panel to complete a cross-sectional survey. Physicians provided information about their practice, general approaches to HD management, and perceptions of available treatment.

Results: A total of 200 neurologists responded to the survey. Based on clinician responses, the most common reasons to treat chorea are impairment in activities of daily living 
$(54 \%)$ and quality of life (41\%). Approximately $50 \%$ of treated patients receive tetrabenazine. More than half of physicians perceive tetrabenazine as having minimal or no effectiveness. In addition to perceptions of insufficient efficacy, $>40 \%$ of physicians agree that tetrabenazine is not optimal owing to side effects or tolerability issues, and $51 \%$ of physicians agree that they are unable to titrate up owing to side effects or tolerability issues. Physicians also report side effects leading to dose interruptions (reported by $33 \%$ of physicians as occurring "often" or "almost always") and reductions (30\%). The most common side effects that led to insufficient dosing and disruptions in titration were sedation/somnolence (41\%), depression (24\%), and anxiety (22\%). In addition, physicians and office staff report spending 1.5 and $2.2 \mathrm{~h}$, respectively, per patient per month managing tetrabenazine-induced side effects.

Conclusions: The study findings emphasize that the practice patterns of physicians who treat HD-related chorea are influenced by tolerability concerns with tetrabenazine, and highlight the need for therapeutic alternatives to tetrabenazine with improved risk-benefit profiles for the treatment of chorea in patients with HD.

\section{Poster \#56}

Treatment Patterns and Unmet Medical Needs in Huntington's Disease in the USA

Daniel O. Claassen ${ }^{1}$, Ravi Iyer ${ }^{2}$, Bijal Shah Manek ${ }^{3}$, Marco DiBonaventura $^{3}$, Victor Abler ${ }^{2}$, Victor Sung ${ }^{4}$

${ }^{1}$ Vanderbilt University Medical Center, Nashville, TN, USA

${ }^{2}$ Teva Pharmaceutical Industries, Frazer, PA, USA

${ }^{3}$ Ipsos Healthcare, San Francisco, CA, USA

${ }^{4}$ University of Alabama School of Medicine, Birmingham, $A L$, USA

\footnotetext{
Abstract

Background: Tetrabenazine, at adjusted doses of up to 100 $\mathrm{mg}$ /day, has shown efficacy for chorea control in patients with Huntington disease's (HD); however, tolerability concerns may lead to insufficient control owing to inability to titrate to optimal doses.

Objectives: To examine pharmacologic treatment patterns, including physician reasons for treatment initiation or noninitiation, and reasons for dose adjustments and discontinuation among patients using tetrabenazine.

Methods: Board-certified/board-eligible neurologists, in practice for $>5$ years and who treated $>3$ patients with HD in the past 2 years were recruited from an online physician panel to complete a retrospective medical chart review of patients with HD with chorea. Physicians provided data on patient disease history, treatment patterns, and healthcare resource use.
}

Results: A total of 138 neurologists responded and 512 patient charts were reviewed. Based on clinician impressions of patients who had used tetrabenazine $(n=377),<40 \%$ experienced a significant improvement ("much better" or "very much better") in their HD chorea after treatment with tetrabenazine. Of patients who had a dose titration from their initial dose $(n=263 ; 70 \%)$, only $56 \%$ achieved optimal chorea control; $31 \%$ were unable to tolerate higher doses and $11 \%$ reached the maximum daily dose without optimal control. The overall average daily stable dose of tetrabenazine was 52.3 $\mathrm{mg}$. In patients who discontinued tetrabenazine $(n=87)$, the most common reasons for discontinuation were lack of efficacy (48\%), patient/caregiver request (39\%), and poor tolerability (38\%). Among patients who never used tetrabenazine $(n=$ 135 ), the most common reasons for not initiating were a history of depression (39\%), reimbursement/access issues (30\%), or a history of anxiety (22\%).

Conclusions: Although some patients with HD achieve chorea control with tetrabenazine, many are unable to tolerate treatment at optimal doses, and others never initiate treatment. These results demonstrate the unmet need for a bettertolerated and efficacious treatment option for chorea associated with HD.

\section{Poster \#57 Invited for Platform Presentation} Update on the LEGATO-HD Study: A Phase II Study Assessing the Efficacy and Safety of Laquinimod as a Treatment for Huntington's Disease

Ralf Reilmann ${ }^{1}$, Mark Forrest Gordon ${ }^{2}$, Karen Anderson ${ }^{3}$, Andrew Feigin $^{4}$, Sarah Tabrizi ${ }^{5}$, Blair Leavitt ${ }^{6}$, Julie Stout ${ }^{7}$, Paola Piccini ${ }^{8}$, Michael Gillespie ${ }^{2}$, Gail Rynkowski ${ }^{2}$, Sarah Swanson $^{2}$, Juha Savola ${ }^{2}$, Spyros Papapetropoulos ${ }^{2}$, Beth Borowsky $^{2}$, Michael Hayden ${ }^{2}$

${ }^{1}$ George-Huntington-Institute Muenster and Department of Neurodegenerative Diseases and Hertie Institute for Clinical Brain Research, University of Tübingen, Tübingen, Germnay ${ }^{2}$ Research and Development, Teva Branded Pharmaceuticals, Vancouver, BC, Canada

${ }^{3}$ MedStar Georgetown University Hospital and Georgetown University Medical Center, Washington, DC, USA

${ }^{4}$ The Feinstein Institute for Medical Research, North ShoreLIJ Health System, Manhasset, NY, USA

${ }^{5}$ UCL Institute of Neurology, London, UK

${ }^{6}$ Centre for Huntington's Disease, University of British Columbia, Vancouver, BC, Canada

${ }^{7}$ Monash University, Melbourne, Australia

${ }^{8}$ Imperial College London, London, UK

\section{Abstract}

Introduction: Laquinimod is an orally active, central nervous system immunomodulator that downregulates inflammatory 
monocytic, microglial, and astrocytic activation; suppresses nuclear factor kappa B activation; and upregulates brain-derived neurotrophic factor, all of which are implicated in the pathological processes in Huntington's disease (HD). The LEGATOHD study was originally designed to assess the efficacy and safety of once-daily doses of laquinimod $0.5,1.0$, and $1.5 \mathrm{mg}$ versus placebo in a 12-month study in patients with HD. Safety concerns with higher doses emerged in a study of patients with multiple sclerosis, and although no safety concern was identified in the LEGATO-HD study, the $1.5-\mathrm{mg}$ arm was discontinued; however, the study continues to evaluate the efficacy and safety of the 0.5 and $1.0 \mathrm{mg}$ laquinimod doses.

Methods: The primary endpoint is change from baseline in Unified Huntington's Disease Rating Scale Total Motor Score (UHDRS-TMS) at month 12; secondary endpoints include safety, brain atrophy, and changes in cognitive and functional capacity. Ancillary studies include microglial activation using positron emission tomography imaging, inflammatory markers, and magnetic resonance spectroscopy analysis.

Results: The study is fully enrolled with 347 patients randomized. As of July 2017, 79 patients have completed and 57 have terminated early (including 30 discontinued from 1.5-mg dose). Baseline mean \pm SD pooled patient demographics (9 June 2017 report) include age $44.4 \pm 7.6$ years, UHDRS-TMS $24.3 \pm 13.3$, UHDRS functional assessment $22.7 \pm 2.5$, UHDRS Total Functional Capacity $11.1 \pm 1.7$, and sex (females 49.3\%). The last patient was enrolled on 18 May 2017. The study is expected to complete patient participation in June 2018.

Conclusions: LEGATO-HD will provide essential data toward possible effective and safe use of laquinimod to reduce the progressive neurodegeneration, and the motor, cognitive, and functional impairments in patients with HD.

\section{Poster \#58}

Predictors for Psychosis in Huntington's Disease: Preliminary Analysis of the Enroll-HD Database Natalia P. Rocha ${ }^{1}$, Erin Furr-Stimming ${ }^{2}$, Antonio L. Teixeira ${ }^{1}$ ${ }^{1}$ Neuropsychiatry Program, Department of Psychiatry and Behavioral Sciences, McGovern Medical School, The University of Texas Health Science Center at Houston, Houston, TX, USA

${ }^{2}$ Movement Disorders \& Neurodegenerative Diseases Program, Department of Neurology, The University of Texas Health Science Center at Houston, Houston, TX, USA

\section{Abstract}

Background: Behavioral problems are present across all stages of HD, even preceding the development of the motor symptoms. While not so frequent in HD, psychosis can be very distressful for both individuals with HD and their caregivers. Psychotic symptoms have been underinvestigated in $\mathrm{HD}$, and their predictors remains to be established.

Objectives: To evaluate predictors for psychosis in HD.

Methods: This study included 2303 patients with manifest HD from the Enroll-HD database (December 2015). We performed univariate analyses in order to investigate differences between patients with HD with and without medical history of psychosis, and all variables that presented a $p$-value $<0.20$ were included in a multivariate analysis (binary logistic regression).

Results: Nearly $11 \%(n=248)$ of patients with HD presented history of psychosis. Among patients with HD with history of psychosis, 55.3\% were premorbid (the age of psychosis symptoms preceded the clinical diagnosis of HD) and $31.6 \%$ had current psychosis (Problem Behaviors Assessment for HD psychosis score $\geq 1$ ). The CAG trinucleotide repeat length was not associated with psychosis. Patients with a history of psychosis presented with a higher frequency of behavioral problems and worse motor, functional capacity, and cognitive scores than patients without a history of psychosis. A logistic regression was performed and patients with HD presenting history of alcohol use disorders, depression, violent/aggressive behavior, preservative/obsessive behavior, and previous suicidal ideation were more likely to exhibit psychosis. Moreover, poorer performance in the Symbol Digit Modalities Test was associated with an increase in the likelihood of psychosis history. The logistic regression model was statistically significant $\left(\chi^{2}=225.755, p=\right.$ 0.000 ), explained $23.0 \%$ of the variance in psychosis history, and correctly classified $90.9 \%$ of cases.

Conclusions: Behavioral problems, previous suicidal ideation, and poorer cognitive performance were significant predictors for psychosis in HD. Psychosis seems to occur in patients with HD that already have a burden of other nonmotor symptoms.

\section{Poster \#59}

Cerebrospinal Fluid Flow Dynamics in Huntington's Disease Using Phase-Contrast Magnetic Resonance Imaging: A Pilot Cross-Sectional Study

Filipe B. Rodrigues ${ }^{1}$, Lauren M. Byrne ${ }^{1}$, Enrico de Vita ${ }^{2}$, Eileanoir Johnson ${ }^{1}$, Rachael Scahill ${ }^{1}$, Edward J. Wild ${ }^{1}$

${ }^{1}$ Huntington's Disease Centre, University College London, $U K$

${ }^{2}$ National Hospital for Neurology \& Neurosurgery, UCLH NHS Trust, London, UK

\section{Abstract}

Objectives: Findings from laboratory research suggest that the flow of cerebrospinal fluid (CSF) may be altered in Huntington's disease (HD). The objective of this study was 
to generate pilot data on CSF flow dynamics in HD using phase-contrast magnetic resonance imaging (PCMRI) to inform the design of future intrathecal drug trials in HD.

Methods: We performed a prospective cross-sectional analysis of 10 age- and sex-matched healthy controls and 10 manifest carriers of HD gene expansion. All participants underwent extensive clinical evaluation and cardiac-gated PCMRI at the level of the Aqueduct of Sylvius, T1 and T10. CSF velocities and flow measurements were derived using a semi-automated method. The influence of age, sex, CAG repeat length, serum osmolality, whole-brain volume, and ventricle volume on these measurements were tested using Spearman correlations or Fisher's exact tests. Group comparisons between healthy controls and manifest carriers were achieved via 2-sample Wilcoxon rank-sum tests. All tests were 2 -sided with a significance level of 0.05 , and corrected for multiple comparisons.

Results: Twenty participants were recruited, and no significant age and sex imbalances were found. None of the studied covariables was found to have an effect on the CSF velocities and flow measurements after corrected for multiple comparisons. No apparent differences were found between study groups in regards to CSF velocities and flow measurements. This pilot study used a novel magnetic resonance imaging technique to study the flow of CSF in 10 people with HD and 10 healthy controls, and found no differences between the groups.

Discussion: Although underpowered, our pilot results add to the view that CSF dynamics are not altered in HD. These results need external validation but offer reassurance that clinically relevant disease-related alterations in CSF flow, which might justify dose-adjustments of intrathecal drugs, are very unlikely to exist. In other words, these results need to be replicated elsewhere but offer reassurance that CSF flow is not altered in $\mathrm{HD}$, which is useful information in planning future trials of drugs that need to be injected into the CSF.

\author{
Poster \#60 \\ Perinatal Insults and Neurodevelopmental Disorders May \\ Impact Age of Diagnosis of Huntington's Disease \\ Melinda Barkhuizen ${ }^{1,2,3}$, Filipe Rodrigues ${ }^{4,5,6}$, David G. \\ Anderson $^{7}$, Edward J. Wild ${ }^{4}$, Boris W. Kramer ${ }^{1,2,8}$, A.W. \\ Danilo Gavilanes ${ }^{1,2,9}$ \\ ${ }^{1}$ Department of Pediatrics, Maastricht University Medical \\ Center (MUMC), Maastricht, The Netherlands \\ ${ }^{2}$ Department of Translational Neuroscience, School of Mental \\ Health and Neuroscience (MHeNs), Maastricht University, \\ Maastricht, The Netherlands \\ ${ }^{3}$ DST/NWU Preclinical Drug Development Platform, North- \\ West University, Potchefstroom, South Africa \\ ${ }^{4}$ Huntington's Disease Centre, Institute of Neurology, \\ University College London, London, UK
}

${ }^{5}$ Clinical Pharmacology Unit, Instituto de Medicina Molecular, Lisbon, Portugal

${ }^{6}$ Laboratory of Clinical Pharmacology and Therapeutics, Faculty of Medicine, University of Lisbon, Lisbon, Portugal ${ }^{7}$ Department of Neurology, University of the Witwatersrand Donald Gordon Medical Centre, Johannesburg, South Africa ${ }^{8}$ School of Oncology and Developmental Biology, Maastricht University, Maastricht, The Netherlands

${ }^{9}$ Institute of Biomedicine, Facultad de Ciencias Médicas, Universidad Católica de Santiago de Guayaquil, Ecuador

\section{Abstract}

Objectives: To determine whether early-life factors, like perinatal insults or neurodevelopmental disorders, are associated with the age of diagnosis of Huntington's disease (HD).

Methods: We used data from 13,863 participants from REGISTRY and Enroll-HD, 2 large international multicenter observational studies. Disease-free survival analyses of mutation carriers with an HTT CAG repeat expansion size $>36$ were computed through Kaplan-Meier estimates of median survival time until a diagnosis of HD. Between groups, comparisons were computed using a Cox proportional hazard survival model adjusted for the CAG-repeat expansion length. All tests were 2-sided with a significance level of 0.05 .

Results: Our results showed that insults in the perinatal period were associated with an earlier median age of diagnosis of 45.00 years [95\% confidence interval (CI) 42.07-47.92) versus 51.00 years $(95 \%$ CI $50.68-51.31)$ in the reference group, with a CAG-adjusted hazard ratio of 1.61 (95\% CI 1.26-2.06). Neurodevelopmental disorders were also associated with an earlier median age of diagnosis of 47.00 years (95\% CI 43.63-50.36) with a CAG-adjusted hazard ratio of 1.41 (95\% CI 1.15-1.73).

Discussion: These results, derived from large observational datasets and using robust survival analysis methods, show that perinatal insults and neurodevelopmental disorders are associated with earlier ages of diagnosis of magnitudes similar to the effects of known genetic modifiers of HD. Given their clear temporal separation, these early events may be causative of earlier HD onset. Even with a survival analysis, this association does prove causation. Further research is needed on the basis of this interaction.

\section{Poster \#61}

Behavioral Testing of Minipigs Transgenic for the Huntington Gene: A 3-Year Follow-Up Study

Verena Schuldenzucker ${ }^{1,2}$, Robin Schubert ${ }^{1}$, Lisa Muratori ${ }^{3}$, Frauke Freisfeld $^{1,4}$, Lorena Rieke ${ }^{1,5}$, Tamara Matheis ${ }^{1,5}$, Sarah Schramke ${ }^{1}$, Nicole Kemper ${ }^{5}$, Ute Radespiel ${ }^{2}$, Ralf Reilmann ${ }^{1,4,6}$ 
${ }^{1}$ George Huntington Institut, Technology-Park, JohannKrane-Weg 27, Muenster, Germany

${ }^{2}$ Institute of Zoology, University of Veterinary Medicine Hannover, Hannover, Germany

${ }^{3}$ Department of Physical Therapy, School of Health Technology and Management, Stony Brook University, Stony Brook, NY, USA

${ }^{4}$ Department of Clinical Radiology, University of Muenster, Muenster, Germany

${ }^{5}$ Institute of Animal Hygiene, Animal Welfare and Farm Animal Behavior, University of Veterinary Medicine Hannover, Hannover, Germany

${ }^{6}$ Department of Neurodegenerative Diseases and HertieInstitute for Clinical Brain Research, University of Tuebingen, Tuebingen, Germany

\begin{abstract}
Background: Huntington's disease (HD) is an autosomal-dominant, progressive neurodegenerative disorder with motor, cognitive, and psychiatric symptoms. So far, there is no curative treatment for HD. Prior to testing novel drugs in human clinical trials, they are tested in preclinical studies to explore safety, tolerability, and efficacy. Large-animal models may provide a more reliable translation into humans because they exhibit a high genetic homology and similar body and brain structure. To test the efficacy of new drugs animal models must replicate symptoms that will target these drugs. We present here the results of a longitudinal and cross-sectional 3-year phenotyping study of a transgenic (tg) HD minipig model.
\end{abstract}

Objectives: To investigate whether tg minipigs develop symptoms of HD during a 3-year period.

Methods: A cohort $(n=32)$ of $\operatorname{tgHD}(n=14)$ and wildtype (wt; $n=18$ ) minipigs were available for all assessments. Tests characterized motor function with a tongue coordination test and hurdle test, cognitive abilities by means of a discrimination test, and the competitive potential by means of a dominance test. The statistical analysis included a repeated analysis of variance to compare the 2 groups over the years and a Wilcoxon test to analyze intravisit differences (or crosssectional differences) between wt and tg minipigs.

Results: After 3 years of behavioral phenotyping, the longitudinal observation of the tests showed a good consistency over the years, a low variance and a good feasibility of the assessments. In some tests, the age of the animals had an influence on their performance, which might reflect developmental and learning effects. No significant differences between $\operatorname{tgHD}$ and wt minipigs have been found.

Conclusions: The assessment battery presented offers a feasible and sensitive set of measures for a continuous phenotyping of the tgHD minipig model or other pig models. If symptoms of a phenotype will develop during the next years, they should be easily detectable with this newly developed test battery.

\section{Poster \#62}

Patient Preferences for Risk-Benefit Assessment of Huntington's Disease

Natasha Scott $^{1}$, Karen Anderson ${ }^{2}$, Erin Wilhelm ${ }^{3}$, Ira Shoulson ${ }^{4}$

${ }^{1}$ Georgetown University, Washington, DC, USA

${ }^{2}$ Medstar Georgetown University Hospital and Georgetown

University, Department of Psychiatry and Department of Neurology, Washington, DC, USA

${ }^{3}$ Georgetown University, Department of Pharmacology \& Physiology, Washington, DC, USA

${ }^{4}$ Georgetown University Medical Center, Department of Neurology, Washington, DC, USA

\section{Abstract}

Background: There is little information available on how patients with Huntington's disease (HD) consider risks and benefits when they compare potential treatment options. This information could inform the design of medical care and research.

Objectives: To explore how patients with HD weigh risks and benefits by learning about their perceived importance of symptoms associated with HD diagnosis.

Methods: Two focus groups of 4 people each were held. The groups were made up of 2 patients with HD, 2 patients with premanifest HD, 2 caregivers, 1 person of unknown genotype, and 1 person who was genotype negative. Participants were asked to state words that came to mind when they, or a family member, first learned about being at risk or affected by HD. These words were used to develop a pipeline of HD symptoms. Each participant was given 9 tokens to distribute among physical, mental, and emotional categories in order to capture how they rated the importance of different symptoms. Participants discussed why particular symptoms were of greatest concern to them.

Results: Overall, participants reported fear and need for more information with receiving a HD diagnosis. Participants commonly worried about how cognitive and emotional symptoms would affect their sense of self and independence, as well as communication with others. Balance issues/falling (24 votes) was rated as the most important physical symptom on which treatment options should be focused, and the most important symptom overall. Slow thinking (17 votes) and lack of emotional regulation (13 votes) were rated as the most important mental and emotional symptoms, respectively.

Conclusions: Currently approved drugs for HD only treat chorea. This study raises the question of whether drug developers and clinicians should focus on other symptoms, given the priority placed on impaired balance, cognition, and emotion. This token allocation approach may assist in understanding how people from families with HD prioritize symptoms of neurodegenerative disease. 


\section{Poster \#63}

Comparative Retrospective Analysis of Tetrabenazine and Olanzapine in Management of Huntington's Disease Based on Data from the Worldwide Observational Study Enroll-HD

Yury Seliverstov ${ }^{1}$, Artyom Borzov ${ }^{2,3}$, Ravil Niyazov ${ }^{5}$, Mikhail Belyaev ${ }^{2,4}$

${ }^{1}$ Department of Neurogenetics, Research Center of Neurology, Moscow, Russian Federation

${ }^{2}$ Institute for Information Transmission Problems (Kharkevich Institute), Moscow, Russian Federation

${ }^{3}$ Moscow Institute of Physics and Technology, Moscow, Russian Federation

${ }^{4}$ Skolkovo Institute of Science and Technology, Moscow, Russian Federation

${ }^{5}$ Center for Scientific Advice Ltd, Moscow, Russian Federation

\begin{abstract}
Background: Only tetrabenazine and deutetrabenazine are approved for treatment of chorea in Huntington's disease (HD). However, a variety of other medicines, including neuroleptics, are used off-label for treating chorea in HD.
\end{abstract}

Objectives: To assess whether data collected during EnrollHD visits are sufficient to study effects of tetrabenazine and olanzapine in HD.

Methods: Of 4752 patients with manifest HD from the third Enroll-HD periodic dataset only 30 patients on tetrabenazine and 17 on olanzapine fulfilled the inclusion criteria. We used the basic inclusion criteria similar to those in the tetrabenazine clinical trial published by the Huntington Study Group in 2006: 1) baseline Unified Huntington's Disease Rating Scale (UHDRS) Total Functional Capacity (TFC) score $>5 ; 2$ ) baseline UHDRS Maximal Chorea score $>9$; 3) treatment duration $\geq 84$ days; 4) every patient used either tetrabenazine or olanzapine; 5) every patient was not taking antichoreatic medication (AM) at the baseline assessment and was on AM at the second assessment, about 1 year later. Basic characteristics of groups were comparable. We analyzed delta for TFC, maximal chorea, maximal dystonia, total motor UHDRS scores along with Problem Behaviors Assessment for HD depression, apathy, and irritability scores between baseline and second visits.

Results: We found a statistically significant improvement of chorea score but worsening of the TFC score in both groups. However, worsening of the TFC score was more pronounced in patients on olanzapine. Interestingly, if restrictions for minimum baseline Maximal Chorea and TFC scores at inclusion are ignored both drugs lose their efficacy for treating chorea. We also found sufficient change in depression score in olanzapine group and in apathy score in tetrabenazine group; however, it did not reach statistically significant levels.

\author{
Poster \#64 \\ Evaluating Wearable Sensors for Objective Measurement \\ of Motor Features of Huntington Disease \\ Jamie Adams ${ }^{1}$, Karthik Dinesh ${ }^{2}$, Mulin Xiong ${ }^{1}$, Christopher \\ Snyder $^{1}$, Christopher A. Tarolli ${ }^{1}$, Saloni Sharma ${ }^{1}$, Nirav \\ Sheth $^{3}$, A.J. Aranyosi ${ }^{3}$, William Zhu ${ }^{1}$, Steven Goldenthal ${ }^{1}$, \\ Kevin Biglan ${ }^{1}$, Ray Dorsey ${ }^{1}$, Gaurav Sharma ${ }^{2}$ \\ ${ }^{1}$ Center for Health and Technology, University of Rochester, \\ Rochester, NY, USA, \\ ${ }^{2}$ University of Rochester, Rochester, NY, USA \\ ${ }^{3}$ MC10 Inc., Lexington, MA, USA
}

\section{Abstract}

Background: Objective, frequent measures of motor function in Huntington's disease (HD) are lacking. The Unified Huntington's Disease Rating Scale (UHDRS) is subjective and generally limited to episodic in-person visits, hindering accurate disease assessment. Wearable sensors, however, can objectively measure motor symptoms both in clinic and at home.

Objectives: To monitor the progression of HD motor symptoms longitudinally using wearable sensors in both in-clinic and at-home settings.

Methods: We enrolled 42 people (15 with HD, 5 with prodromal HD, 22 controls without a movement disorder) and asked them to wear 5 accelerometer-based MC10 BioStampRC sensors on their chest and limbs. Over a 12-month period, we are collecting data through both in-clinic and at-home assessments. We want to compare sensor results between HD, prodromal $\mathrm{HD}$, and control individuals, as well as track the progression of HD motor symptoms using novel sensor-derived metrics.

Results: We developed a classifier that can predict when an individual is lying down, standing up, sitting, or walking. Results from the baseline show individuals with HD spending significantly more time lying down than controls $(50.1 \%$ vs $34.0 \% ; p<0.001)$. Preliminary longitudinal results corroborate this pattern. We were able to objectively rate the degree of gait regularity using cross-correlation between the left and right leg sensors and distinguish between individuals with HD and controls using step duration. Longitudinal comparison between the first and second visits show that step duration increased in individuals with HD over a roughly 6-month period. Preliminary results based on longitudinal data also suggest individuals with HD undergo fewer sit-to-stand transitions than controls. 
Conclusions: We objectively measured gait-related motor symptoms of HD and identified notable differences in lifestyle between individuals with $\mathrm{HD}$ and controls using a lightweight wearable sensor system.

Sponsorship: This study is sponsored by MC10.

\section{Poster \#65}

\section{Cerebrospinal Fluid Mutant Huntingtin Concentration:} What Does it Mean?

Nicholas Caron ${ }^{1}$, Christopher Yanick $^{2}$, Stephen E.P. Smith ${ }^{3}$, Yuan-Yun Xie ${ }^{1}$, Ji-Joon Song ${ }^{4}$, Ihn Sik Seong ${ }^{5}$, Blair R. Leavitt $^{1}$, Michael R. Hayden ${ }^{1}$, Amber L. Southwell ${ }^{2}$

${ }^{1}$ Center for Molecular Medicine and Therapeutics, University of British Columbia, Vancouver, Canada

${ }^{2}$ Burnett School of Biomedical Sciences, University of Central Florida, Orlando, FL, USA

${ }^{3}$ Seattle Children's Research Institute, University of Washington, Seattle, WA, USA

${ }^{4}$ Department of Biological Sciences, KAIST, Daejeon, Korea

${ }^{5}$ Department of Neurology, Massachusetts General Hospital, Boston, MA, USA

\footnotetext{
Abstract

Background: A variety of huntingtin (HTT)-lowering strategies have shown preclinical promise for the treatment of Huntington's disease (HD), and the first HTT-lowering clinical trial is now underway. Based on our previous work demonstrating that central nervous system (CNS)-wide suppression of HTT in mice results in correlative reduction of cerebrospinal fluid (CSF) mtHTT, CSF mtHTT is being used as a marker of target engagement. However, interpretation of treatment-induced changes, or lack thereof, is not currently feasible.

Methods: We are using ultrasensitive immunoprecipitation and flow cytometry mutant HTT (mtHTT) detection to enable substantive use of CSF mtHTT as an HD biomarker. Specifically, unlike mice, HTT lowering in humans is not expected to be CNS-wide, but partial regional reduction may be insufficient to result in measureable CSF reduction. This could erroneously indicate failure to suppress HTT. Thus, we are interrogating the source of CSF mtHTT using BACHD mice (floxed mtHTT exon 1) crossed to brain region and cell type-specific cre mice to generate mice with selective knockout of mtHTT and to assess the contribution of brain regions and/or cell types to the pool of CSF mtHTT. Additionally, the mechanism of mtHTT entry into CSF is unknown. We have previously shown that mtHTT is not detected in CSF of all premanifest HD mutation carriers, and that acute brain injury in mice causes a transient increase in CSF mtHTT, suggesting that HTT is released to CSF from dying cells. However, we also detect mtHTT in CSF of mice that lack neurodegeneration, suggesting that there may be both passive release and active clearance mechanisms involved. By ectopically
}

delivering intracellular and extracellular mtHTT to the brains of mice, we are investigating the mechanism of mtHTT clearance to CSF.

Conclusions: Delineating the source and mechanism of entry of CSF mtHTT protein will greatly enhance the utility of this measure as an HD biomarker.

\section{Poster \#66}

Feasibility of Smartphone Application Use for the Objective Evaluation of the Effects of Huntington's Disease on Motor Function

Kelsey L. Spear, Jamie L. Adams, Molly J. Elson, Tyler S. Geery, Michael J. Curtis, David J. Mitten, E. Ray Dorsey University of Rochester Medical Center, Rochester, NY, USA

\section{Abstract}

Background: As technology becomes increasingly inexpensive, ubiquitous, and advanced, smartphones present a unique opportunity to measure disease. Previous studies of applications have demonstrated that smartphones effectively measure key characteristics of Parkinson's disease, sensitively recognize daily variability and response to medication, and strongly correlate with the Unified Parkinson Disease Rating Scale.

Objectives: To assess the feasibility of using an Android smartphone application (app) to quantitatively measure factors of Huntington's disease (HD), such as gait, chorea, voice, balance, dexterity, bodily motion, mobility, and socialization.

Methods: We are testing an app, called George, that has the potential to quantify motor symptoms, distinguish between individuals with and without $\mathrm{HD}$, and assess the response to drug treatment. By more frequently monitoring HD symptoms through the use of smartphone application measurements, we can better detect changes in symptoms. The app utilizes the phone's sensors to assess chorea and balance, the screen to assess tapping speed, the microphone to assess voice, and the GPS to assess socialization. By collecting more data in shorter periods of time, this technology could expedite research studies and the development of novel therapeutics for HD, and advance exploratory clinical trials to assess HD. Remote data collection will also ease the time and travel burden for research participants and caregivers. We are conducting an observational study of 40 participants, 20 to 25 with clinically diagnosed HD, 5 to 10 with prodromal HD, and 10 controls without a movement disorder. Participants will undergo 2 in-clinic assessments, as well as complete the app's tasks daily for 1 month. The primary outcome measures include feasibility, quantitative motor, and socialization data.

Conclusions: This pilot study will result in further refinement of the application based on participant feedback and data collected, and ideally lay the foundation for its use in evaluating the efficacy of current and future therapies for HD. 


\section{Poster \#67}

Tracking Huntington's Disease in Italian Premanifest and Manifest Subjects by Connecting REGISTRY and EnrollHD Observational Studies

Ferdinando Squitieri $^{1}$, Sabrina Maffi ${ }^{1}$, Simone Migliore ${ }^{1,2}$, Massimo Marano ${ }^{1,2}$, Irene Mazzante ${ }^{2}$, Iolanda Santimone ${ }^{1}$, Francesca Lovo ${ }^{2}$, Barbara D’Alessio ${ }^{2}$

${ }^{1}$ Huntington and Rare Diseases Unit, IRCCS Casa Sollievo della Sofferenza Hospital (San Giovanni Rotondo), CSSMendel, Rome, Italy

${ }^{2}$ LIRH Foundation, Rome, Italy

\begin{abstract}
Background: Monitoring Huntington's disease (HD) longitudinal clinical changes is the main aim of the observational studies. The European REGISTRY in its version 1, 2, and 3 and the global Enroll-HD represent a crucial opportunity to track the HD development during time, with all the many variables potentially influencing it, by either environmental or biological factors. The Italian League for Research on Huntington and related diseases, LIRH-Foundation (named LIRH-Rome site), started REGISTRY in May 2005 and Enroll-HD in July 2014.
\end{abstract}

Methods: In about 12 years, we have recruited about 330 subjects in REGISTRY and 500 in Enroll by February 2017. We have now started to track the disease course of patients who started REGISTRY and were able to continue into Enroll. Some of these patients were followed-up for many years. We followed-up 165 REGISTRY subjects and 283 Enroll subjects from pre-manifest to advanced HD stage.

Results: REGISTRY's subjects who were disease tracked since REGISTRY showed a maximum yearly observation number of 12 years (range 2-12), while maximum number of yearly observations in Enroll was 4. The Total Functional Capacity Scale score ranged between 13 and 0 (mean 7.8), and the Total Motor Score ranged between 0 (premanifest) and 118 (advanced stage). The mean expanded CAG repeat number was 44.1, ranging between 36 and 87 (those with $>60$ repeats showing infantile/adolescent age at onset). We also shared the cohort according to the geographical area where they live (north, mid, and southern Italy) to track difference in score changes according to the environment.

Conclusions: Monitoring HD longitudinally is a crucial step to disclose differences in HD development in homogenous ethnic populations to highlight environmental and biological influence over the disease progression.

\section{Poster \#68}

Efficient Genome Editing in the Mouse Brain by Local Delivery of Engineered Cas9

Brett T. Staahl ${ }^{1}$, Madhurima Benekareddy ${ }^{2}$, Claire CoulonBainier $^{2}$, Ashwin A. Banfal ${ }^{1}$, Stephen N. Floor ${ }^{1}$, Jennifer K.
Sabo ${ }^{1}$, Cole Urnes ${ }^{1}$, Gabriela Acevedo Munares ${ }^{1}$, Anirvan Ghosh $^{2,3}$, Jennifer A. Doudna ${ }^{1,4,5,6,7}$

${ }^{1}$ Department of Molecular and Cell Biology, University of California, Berkeley, CA, USA

${ }^{2}$ Roche Pharma Research and Early Development, Basel, Switzerland

${ }^{3}$ E-Scape Bio, San Francisco, CA, USA

${ }^{4}$ Howard Hughes Medical Institute, University of California, Berkeley, CA, USA

${ }^{5}$ Innovative Genomics Institute, University of California, Berkeley, CA, USA

${ }^{6}$ MBIB Division, Lawrence Berkeley National Laboratory, Berkeley, CA, USA

${ }^{7}$ Department of Chemistry, University of California, Berkeley, CA, USA

\section{Abstract}

Background: The CRISPR system is a gene-editing tool that uses the enzyme Cas9, a type of molecular scissors, to cut out a particular gene. The strategy could overhaul the treatment of genetic diseases by replacing or deleting problematic genes with functional ones. However, for it to work, a gene for Cas9 is inserted into cells, and continuous expression of this gene may cause problems; it could alter other genes, insert itself into the cell's DNA, and, over time, cause an immune response.

Methods: We demonstrate editing of postmitotic neurons in the adult mouse brain following injection of Cas9 ribonucleoprotein complexes in the hippocampus, striatum, and cortex. Engineered variants of Cas9 with multiple SV40 nuclear localization sequences enabled a 10-fold increase in the efficiency of neuronal editing in vivo. When injected into the brains of mice it deleted a segment of DNA in neurons. Unlike genetically encoded solutions, this Cas 9 rapidly degraded, preventing long-term consequences.

Results/conclusions: These advances indicate the potential of genome editing in the brain to correct or inactivate the underlying genetic causes of neurological diseases. Development of a gene-editing technique reduces some of the risks associated with the process, moving the field one step closer to a safe treatment for the underlying cause of Huntington's disease.

\section{Poster \#69}

Microglia Mediate Early Loss of Specific Synaptic Connections in Huntington's Disease

Daniel K. Wilton ${ }^{1}$, M. Heller ${ }^{1}$, Anthony Daggett ${ }^{2}$, Arthur Y. Kim $^{1}$, A. Frouin ${ }^{1}$, Marika Eszes ${ }^{3}$, Richard Faull ${ }^{3}$, W.X. Yang $^{2}$, Beth Stevens ${ }^{1}$

${ }^{1}$ Department of Neurology, Boston Children's Hospital, Harvard Medical School, Boston, MA, USA 
${ }^{2}$ Center for Neurobehavioral Genetics, Semel Institute for Neuroscience and Human Behaviors, David Geffen School of Medicine at University of California, Los Angeles, CA, USA

${ }^{3}$ The University of Auckland, Grafton, Auckland, New Zealand

\begin{abstract}
Background: Synaptic dysfunction is increasingly recognized as one of the earliest pathological events in many neurodegenerative diseases, including Huntington's disease (HD). Despite HD being a monogenic disease with a well-characterized pathology, the molecular mechanisms underlying synaptic dysfunction remain poorly understood. In this study, we show that loss of specific synaptic connections can be observed as early as 3 months in multiple slow progressive HD mouse models prior to the onset of motor or cognitive impairment and that microglia contribute to this via a complementdependent mechanism.
\end{abstract}

Methods/results: During development, synapse loss as a result of pruning is a normal and highly regulated process required for correct wiring of the brain. Microglia together with complement proteins, a key component of the innate immune system, play a key role in regulating this process. Here, we provide evidence that aspects of this developmental mechanism are aberrantly reactivated in HD mouse models, leading to the loss of specific synaptic inputs. Using super-resolution imaging, genetic ablation, and viral-mediated labeling, we demonstrate that complement proteins selectively target corticostriatal connections for engulfment by microglia and that this process requires mutant huntingtin expression in both striatal and cortical neurons. Moreover, blocking this process at multiple levels through both genetic- and antibodymediated approaches is able to prevent synaptic pathology. Notably, we find that aspects of this mechanism may be conserved in human disease, as postmortem tissue reveals evidence of synapse loss, complement deposition at synapses, and phagocytic microglia. Together, these findings demonstrate that elements of the innate immune system previously only thought to be involved in late-stage inflammation mediate the loss of specific synaptic connections early in HD pathology.

\section{Poster \#70}

"I Have a Feeling I Can't Speak to Anybody": A Thematic Analysis of Communication Perspectives in People with Huntington's Disease

Nicolò Zarotti, Jane Simpson, Ian Fletcher

Division of Health Research, Faculty of Health and Medicine,

Lancaster University, Lancaster, UK

\section{Abstract}

Background: The current literature on communication in Huntington's disease (HD) appears to be mainly characterized by quantitative studies focused on the observation of medical and cognitive impairments, whereas the investigation of the patients' subjective experiences is generally much rarer. This study explored the perspectives of people affected by HD on their own communicative abilities.

Methods: Qualitative semi-structured interviews were carried out with 8 people with early-stage HD. The data were analyzed through thematic analysis. Four themes were constructed from the resulting data, characterized by the following core topics: how HD directs and mediates communication; regaining control to improve communication; emotional outflows into communication and the struggle for separation; and sheltering as a way to boost confidence in communication.

Results: The findings showed that separating patients' identity as individuals from that of a person with a disease can help increase communicative control. Consistent with the general theory and model of self-regulation, patients should be allowed a wider range of choices to regain control over communication. In addition, achieving better emotion regulation is of paramount importance for communication, and factors such as medication regimes, relationships, and existing coping strategies should be strengthened. Consistent with previous research, feelings of safety and the idea of a safe place ("sheltering") represent an effective coping mechanism. Practical implications include the refinement of communication and relationships among clinicians, caregivers, and patients with HD by considering a wider range of medical, psychological, and socio-environmental factors.

Poster \#71 Award Winner for Michael Conneally Genetics Abstract

Selection of an Adeno-Associated Virus Gene Therapy Targeting Huntingtin for the Treatment of Huntington's Disease

Pengcheng Zhou ${ }^{1}$, Fen $\mathrm{Chen}^{2}$, Xin Wang ${ }^{1}$, Emily Christensen $^{1}$, Jeff Thompson ${ }^{1}$, Mathieu Nonnenmacher ${ }^{1}$, Maria Scheel $^{1}$, Xiao Ren ${ }^{1}$, Wei Wang ${ }^{1}$, Xiaochuan Zhou ${ }^{1}$, Lisa Stanek ${ }^{2}$, Bryan Mastis ${ }^{2}$, Peter Pechan ${ }^{1}$, Eric Horowitz ${ }^{1}$, David Dismuke ${ }^{1}$, Adrian Kells ${ }^{1}$, Todd Carter ${ }^{1}$, Jay Hou ${ }^{1}$, Dinah $\mathrm{Sah}^{1}$

${ }^{1}$ Voyager Therapeutics, Inc., Cambridge, MA, USA

${ }^{2}$ Sanofi/Genzyme, Framingham, MA, USA

\section{Abstract}

Background: Studies in animal models of Huntington's disease (HD) support a strategy focused on lowering huntingtin (HTT) mRNA and protein, as partial suppression of HTT in the central nervous system is both safe and 
effective. Adeno-associated virus (AAV) gene therapy with RNA interference (RNAi) targeting HTT mRNA selectively for degradation represents such a strategy and has the potential to provide therapeutic benefit. Here, we describe studies to select the clinical candidate VY-HTT01 comprising an AAV gene therapy targeting HTT with RNAi for the treatment of HD.

Methods: All components of the AAV gene therapy targeting HTT with RNAi were optimized for potency and selectivity, including the capsid and vector genome comprising the primary microRNA (pri-miRNA, including cassette and RNAi sequence), promoter, and configuration of components. Approximately 120 pri-miRNAs and vector genome configurations were screened in vitro using 6 human cell lines, including fibroblasts from patients with HD. Sixteen candidates targeting HTT were selected for comparison in YAC128 transgenic mice expressing human mutant HTT. Based on human HTT mRNA knockdown in the striatum, and precision and efficiency of pri-miRNA processing, 4 candidates were selected for the lead selection study in nonhuman primates. VYHTT01 was chosen based on HTT lowering in the putamen, precision and efficiency of pri-miRNA processing, and safety in the nonhuman primate.

Disclosures: These studies were supported, in part, by the Cure Huntington's Disease Initiative.

\section{Poster \#72}

A Pilot Study Evaluating Plasma Endocannabinoid Levels as Biomarkers in Huntington's Disease

Grace Zimmerman ${ }^{1}$, Peter Morrison ${ }^{1}$, Kevin Welle ${ }^{1}$, Jennifer Hryhorenko ${ }^{1}$, Stephen D'Ambrosio ${ }^{1}$, Iris Young ${ }^{1}$, Michael McDermott $^{1}$, Kevin Biglan ${ }^{1}$, Kelly Andrzejewski ${ }^{1,2}$

${ }^{1}$ Department of Neurology, University of Rochester, Rochester, NY, USA

${ }^{2}$ Department of Neurology, University at Buffalo, Buffalo, NY, USA

\footnotetext{
Abstract

Background: Cannabinoid 1 (CB1) receptors are decreased in the basal ganglia in early stages of Huntington's disease (HD), even before motor symptom manifestations of the disease appear. Cannabinoid 2 (CB2) receptors present on activated microglia in the brain are also observed postmortem in HD. The endocannabinoids anandamide (AEA) and 2arachidonoyl glycerol (2-AG) are involved in the regulation of medium spiny neurons and microglial inflammation by acting at $\mathrm{CB} 1$ receptors in the basal ganglia, and at $\mathrm{CB} 2$ receptors on microglia.

Objectives: To evaluate if plasma and cerebrospinal fluid (CSF) levels of the endocannabinoids AEA and 2-AG were different in HD participants versus healthy controls, and
}

whether they were correlated with measures of HD symptom severity and cognition.

Methods: Blood was collected from controls and patients with HD to measure AEA and 2-AG levels using ultraperformance liquid chromatography with mass spectrometry. The Unified Huntington's Disease Rating Scale (UHDRS) Total Motor Score (TMS) and Total Functional Capacity (TFC) score, and Montreal Cognitive Assessment (MoCA) were completed for individuals with HD.

Results: There were no significant differences in plasma AEA and 2-AG levels between healthy controls $(n=6)$ and participants with $\mathrm{HD}(n=8)$. Although not significant, mean total plasma 2-AG level was lower in individuals with HD $(2.43 \pm$ 1.02) than in controls $(9.29 \pm 13.12)(p=0.18)$. AEA level was correlated with UHDRS TFC score $(\mathrm{r}=0.61, p=0.11)$. No other correlations between plasma endocannabinoid levels and UHDRS TMS, total chorea subscore, TFC, or MoCA were observed. Neither AEA nor 2-AG levels were detected in CSF.

Conclusions: Changes in the endogenous cannabinoid system in HD may hold potential to serve as markers of disease state. However, a future study evaluating plasma endocannabinoid levels in a larger number of participants will be required owing to the small sample size and high interparticipant variability in plasma endocannabinoid levels in our study. Changes in the endogenous cannabinoid system in the brain are observed several years before individuals develop the motor symptoms of HD. We evaluated levels of endocannabinoids in the blood of both healthy controls and individuals with HD, and did not observe any significant differences in levels of two of these endocannabinoids.

\section{Poster \#73 \\ Genotype-Phenotype Correlation of Huntingtin CAG Repeat Expansion and Associated Symptoms in a Large Indian Family Carrying Huntington's Disease Mutation Roshni Kishor Bhatt1, Sudhir V. Shah2, Hitarthi Vyas1, Ravi Vijayvargiyal \\ ${ }^{1}$ Department of Biochemistry, The Maharaja Sayajirao University of Baroda, Vadodara India \\ ${ }^{2}$ Department of Neurology, Seth K M School of Postgraduate Medicine and Research, Ahmedabad, India}

\begin{abstract}
Background:We describe a 47-year-old man with a 20-year history of behavioral changes, chorea, and hallucinations. History and mental state examination were suggestive of Huntington's disease (HD). Neurological examination revealed discrete, involuntary movements affecting his arms and trunk. Genotyping detected an expanded allele (43 CAG repeats, other allele $17 \mathrm{CAG})$. A detailed investigation
\end{abstract}


revealed a three-generation-long family history of chorea and psychosis. There was a family history of HD in a sibship of 12 individuals, nine of whom were older than him. The origin of the mutation was traced to an ancestral paternal allele. This is a case study to look at an increased expansion in a family where the paternal allele of 43 CAG repeats expanded in the next generation to an allele with 47 repeats.

Methods: To determine the CAG expansion, blood samples were obtained after informed consent, and CAG repeat length was determined by PCR analysis. Samples from five family members, including this patient, were processed. To evaluate the impact of CAG repeat expansion on disease progression and symptoms, the Mini-Mental State Examination (MMSE) was carried out in these subjects

Results: All five family members were found to carry the HD mutation with the CAG repeat expansion in the affected allele ranging from 43-47. The comparison between age of onset and duration of HD resulted in a Pearson correlation coefficient (r) value of 0.8944 , and MMSE had a probability value of 0.010769. Investigation of correlation with CAG repeat size is underway.

Conclusions: In the current study, we have identified 12 living members of a large Indian family with symptoms of HD. Five patients were confirmed to carry the HD mutation by genetic testing. Remaining members will also be tested, and a detailed genotype-phenotype analysis will be carried out.

\section{Poster \#74}

Molecular and Cellular Assessment of HTT Lowering in Differentiated Patient-Derived HD iPS Cells

Lisa L. Salazar ${ }^{1}$, Ryan G. Lim² ${ }^{2}$ Andrea M. Reyes-Ortiz ${ }^{2}$, Sarah J. Hernandez ${ }^{3}$, Charlene Geater ${ }^{1}$, Austin Hill ${ }^{4}$, Julia A. Kaye ${ }^{5}$, Jennifer T. Stocksdale ${ }^{6}$, Keona Wang ${ }^{3}$, Lexi K. Kopan $^{3}$, Steven Finbeiner ${ }^{5,7}$ Blair Leavitt4, Leslie M. Thompson ${ }^{1,2,3,6,8}$

${ }^{1}$ Department of Psychiatry and Human Behavior, University of California, Irvine, Irvine, California, USA.

${ }^{2}$ Department of Biological Chemistry, University of California, Irvine, Irvine, California, USA.

${ }^{3}$ Department of Neurobiology and Behavior, University of California, Irvine, Irvine, California, USA.

${ }^{4}$ Department of Medical Genetics, Centre for Molecular Medicine and Therapeutics, Child and Family Research
Institute, University of British Columbia, Vancouver, BC, Canada V5Z 4 H4.

${ }^{5}$ Gladstone Institutes and the Taube/Koret Center of Neurodegenerative Disease Research, Roddenberry Stem Cell Research Program, San Francisco, California, USA.

${ }^{6}$ UCI MIND, University of California, Irvine, Irvine, California, USA.

${ }^{7}$ Departments of Neurology and Physiology, University of California, San Francisco, San Francisco, California, USA.

${ }^{8}$ Sue and Bill Gross Stem Cell Center, University of California, Irvine, Irvine, California, USA..

\begin{abstract}
Background:The current clinical trial of the total Huntingtinlowering ASO (IONIS-HTTRx) has generated excitement in both the HD field and patient communities. Preclinical mouse studies show behavioral improvement and support general safety, and preliminary results from completed cohorts of the safety (phase1/2a) trial indicate a favorable safety and tolerability profile. Once the analysis of the trial is completed, the next step may be an efficacy trial testing whether IONISHTTRx can slow progression of the disease. Indeed, an area not fully addressed in the field is the specific, and potentially complex, alterations to normal neuronal function and gene expression when total HTT is reduced. Further, the differential effects of preferentially reducing mutant HTT (through allele preferential approaches) compared to total HTT knockdown have not been systematically examined.
\end{abstract}

Methods: To begin to address these questions, we are evaluating the potential of total versus mutant HTT lowering to correct cellular and gene expression changes in HD iPSCderived neurons. Control and HD iPSC lines have been generated to reduce total or mutant HTT in an inducible manner and preliminary evaluations indicate successful induction of HTT lowering. We are also determining the effects of total HTT lowering following treatment with ASOs or siRNA delivered using a highly effective lipid nanoparticle system. Specific functional and molecular readouts are in progress to evaluate the consequence of HTT-lowering approaches, and will be presented.

Conclusions: : Results from these studies will further inform clinical HTT-lowering treatments regarding the safety and efficacy of targeting HTT. 